OPEN ACCESS

Edited by:

Yue Liu,

Xiyuan Hospital, China

Reviewed by:

Xing Liao,

China Academy of Chinese Medical

Sciences, China

Wiejian Bei,

Guangdong Pharmaceutical

University, China

Yonggang Zhang,

Sichuan University, China

${ }^{*}$ Correspondence:

Jiarui Wu

exogamy@163.com

Specialty section: This article was submitted to

Ethnopharmacology,

a section of the journal

Frontiers in Pharmacology

Received: 25 May 2020

Accepted: 30 July 2020

Published: 26 August 2020

Citation:

Guo S, Wu J, Ni M, Jia S, Zhang J, Zhou W, Liu X, Wang M and Zhang $X$

(2020) Comparative Efficacy of Danshen Class Injections for Treating Acute Coronary Syndrome: A

Multidimensional Bayesian Network

Meta-Analysis of Randomized

Controlled Trials.

Front. Pharmacol. 11:1260.

doi: 10.3389/fphar.2020.01260

\section{Comparative Efficacy of Danshen Class Injections for Treating Acute Coronary Syndrome: A Multidimensional Bayesian Network Meta-Analysis of Randomized Controlled Trials}

Siyu Guo, Jiarui Wu*, Mengwei Ni, Shanshan Jia, Jingyuan Zhang, Wei Zhou, Xinkui Liu, Miaomiao Wang and Xiaomeng Zhang

Department of Clinical Chinese Pharmacy, School of Chinese Materia Medica, Beijing University of Chinese Medicine,
Beijing, China

Background: Acute coronary syndrome, that is a common and serious cardiovascular disease, imposes a huge economic burden on global public health. And Danshen class injections are commonly used in the treatment of acute coronary syndrome in China. Thus, the Bayesian network meta-analysis was devised to investigate the efficacy of different Danshen class injections against acute coronary syndrome.

Methods: Eligible inclusion and exclusion criteria were established in advance. Then, a systematic literature search was performed in several databases from inception to February 2020. Further, the included randomized controlled trials data were adopted to calculation, prepare graphs and multidimensional cluster analysis by WinBUGS 1.4.3, Stata V.13.0 and R 3.6.1 software, respectively.

Results: A total of 53 eligible randomized controlled trial studies with 6401 patients were obtained that evaluated the clinical effectiveness rate, the level of hypersensitive C-reactive protein, C-reactive protein, interleukin-6, fibrinogen, and adverse reactions after the application of Danshen class injections plus western medicine. Compared with western medicine alone, Danshen class injections combined with western medicine therapy were associated with significantly improved the therapeutic effect. In addition, the results of the multidimensional cluster analysis demonstrated that Danhong injection + western medicine and Danshen injection + western medicine had better therapeutic effects. However, since most eligible randomized controlled trial studies did not focus on the monitoring of adverse reactions, the safety of these Chinese herbal injections needs to be further explored.

Conclusion: Based on this Bayesian network meta-analysis results, Danhong injection + western medicine and Danshen injection + western medicine might have a better impact on acute coronary syndrome patients. Nevertheless, more large samples, high-quality 
clinical and multicenter randomized controlled trial studies should be tested and verified in the future.

Keywords: Danshen class injections, acute coronary syndrome, network meta-analysis, randomized controlled trials, outcomes research

\section{INTRODUCTION}

Acute coronary syndrome (ACS) is a medical emergency (Kosuge and Kimura, 2016). The underlying mechanism of ACS is triggered by atheromatous plaque enlargement, instability, and rupture or erosion (Amsterdam et al., 2014; Saremi, 2017). ACS includes ST elevation myocardial infarction (STEMI), unstable angina (UA) and non-ST elevation myocardial infarction (NSTEMI) (Hedayati et al., 2018). In 2014, it is estimated that in the United States, 633,000 persons were diagnosed with acute coronary syndrome (Virani et al., 2020). Additionally, the risk of ACS is greater with age (Timmis, 2015). Presently, the treatments for ACS mainly rely on anti-platelet, anti-coagulant, anti-ischemic, thrombolytic and percutaneous coronary intervention (PCI) (Wu et al., 2015). However, although clopidogrel is an effective antiplatelet drug in patients with ACS, it has the limitations of high bleeding risk and stent thrombosis (Wang et al., 2018). Previous studies reported that PCI may be related to vascular endothelial cell dysfunction, platelet aggregation and neutrophil obstruction, which will lead to restenosis of the stent or some major adverse cardiac events (MACE) (Farooq et al., 2011; Kirtane et al., 2014).

To find more effective and promising therapeutic approaches to manage ACS, traditional Chinese medicine (TCM) combined with western medicine (WM) have received widespread attention from clinicians in China (Zhang et al., 2020). In the theory of TCM, the pathogenesis of ACS is closely correlated to stagnant blood block (Wu et al., 2015). Furthermore, Chinese herbal injections (CHIs) are a new type of TCM formulation with high bioavailability and fast action (Li et al., 2017). According to accumulated evidenced-based data, the combination of CHIs and WM is associated with considerable efficacy in treating patients with ACS (Zhao et al., 2018). For example, a systematic review reported that Danhong injection $(\mathrm{DH})$ could improve the therapeutic efficacy and reduce the incidence of

\footnotetext{
Abbreviations: ACS, acute coronary syndrome; ADRs, adverse reactions; CHIs, Chinese herbal injections; CNKI, the China National Knowledge Infrastructure Database; CRP, C-reactive protein; DH, Danhong injection; DS, Danshen injection; DSCIs, Danshen class injections; DSCXQ, Danshenchuanxiongqin injection; DSDFSY, Dansenduofensuanyan injection; DSSA, Danshen Salvianolic Acids injection; FFDS, Fufang Danshen injection; FIB, fibrinogen; FIB-C, fibrinogen C; GXN, Guanxinning injection; hs-CRP, hypersensitive C-reactive protein; IL-6, interleukin-6; MACE, major adverse cardiac events; MDs, mean differences; NF- $\kappa B$, nuclear factor- $\kappa B$; NMA, network meta-analysis; NSTEMI, non-ST elevation myocardial infarction; ORs, odds ratios; PCI, percutaneous coronary intervention; PRISMA, the Preferred Reporting Items for Systematic Reviews and Meta-Analyses; RCTs, randomized controlled trials; SinoMed, the Chinese Biomedical Literature Database; STEMI, ST elevation myocardial infarction; STS, Sodium Tanshinone IIA Sulfonate injection; SUCRA, surface under the cumulative ranking; TCM, traditional Chinese medicine; UA, unstable angina; VIP, the Chinese Scientific Journals Full-text Database; WM, western medicine.
}

MACE events in ACS patients after PCI (Zou et al., 2018). Moreover, systematic reviews and meta-analysis studies shown that Sodium Tanshinone IIA Sulfonate injection (STS) and Danshenchuanxiongqin injection (DSCXQ) combined with WM appeared to be efficacious in the treatment UA (Zhang et al., 2016; Tan et al., 2018).

In China and Japan, Danshen (Radix Salviae Miltiorrhizae) is the dried root or rhizome of Salvia miltiorrhiza Bunge, commonly used to treat cardiovascular-related diseases (Meim et al., 2019; Yuan et al., 2020). The relative studies validated that Danshen possesses the characteristics of relaxing coronary artery muscle, improving microcirculation, reducing blood viscosity and inhibiting thrombogenesis (Cheng, 2006). The CHIs containing the extract of Danshen are termed Danshen class injections (DSCIs) (Liu et al., 2019). In this context, this study included 8 DSCIs, namely, Danshen injection (DS), Fufang Danshen injection (FFDS), Danhong injection (DH), Dansenduofensuanyan injection (DSDFSY), Danshen Salvianolic Acids injection (DSSA), Danshenchuanxiongqin injection (DSCXQ), Sodium Tanshinone IIA Sulfonate injection (STS) and Guanxinning injection (GXN). Compared with the traditional double-arm meta-analysis, network meta-analysis (NMA) can synthesize different interventions for the same disease and perform direct or indirect comparisons (Grant, 2019; Sun et al., 2019; Zhang et al., 2019). Moreover, NMA can assist assessing and ranking the therapeutic effects of different treatments (Rouse et al., 2017; Pan et al., 2019). However, at present, there are many systematic review studies on the adjuvant treatment of ACS with a certain type of DSCIs, lacking of comparison of efficacy between different types of DSCI (Wu et al., 2015; Zhang et al., 2016; Wu et al., 2017; Tan et al., 2018; Zou et al., 2018). Therefore, the present research adopted NMA to explore the comparative effectiveness and safety between different DSCIs plus WM against ACS, providing a reference for clinical practice.

\section{MATERIALS AND METHODS}

The procedure of the current NMA research was conducted based on the Preferred Reporting Items for Systematic Reviews and Meta-Analyses (PRISMA) guidelines (Hutton et al., 2015) (Supplementary File 1).

\section{Eligibility and Exclusion Criteria}

The Eligibility criteria for this study conformed the PICOS framework, namely, participants, interventions, comparisons, outcomes and study design. So, randomized controlled trials (RCTs) that met the following criteria were included in this 
study: (1) Study design. Only RCTs mentioned in articles were enrolled. (2) Participants: The current NMA included patients were diagnosed with ACS, and without limitations on race, age, gender, or nationality. (3) Interventions and comparisons: The experiment group was treated by CHIs (DS, FFDS, DH, DSDFSY, DSSA, DSCXQ, STS, and GXN) combined with WM. Patients in the control group were both received WM treatment or WM plus another CHIs, excluding PCI. The commonly used WM drugs were primarily nitrate, low molecular weight heparin, aspirin, nifedipine, statins, $\beta$-receptor blocker, and so on. There are no restrictions on dosage and duration of treatment. The corresponding treatment measures should be taken for patients with other complications. (4) Outcomes. The primary outcomes of this study were the clinical effectiveness rate. According to clinical symptoms and objective indicators, the effectiveness status was divided into effective, effective, and ineffective. The clinical effectiveness rate $=$ (number of total patients - number of invalid patients)/number of total patients $* 100 \%$. It was regarded as invalidation when the clinical symptoms and electrocardiogram didn't change or worsen, or the frequency of chest pain wasn't decreased or increased (Ministry of Health of People's Republic of China, 1993). Additionally, the secondary outcomes were hypersensitive C-reactive protein (hsCRP), C-reactive protein (CRP), interleukin-6 (IL-6), fibrinogen (FIB) and adverse reactions (ADRs). Previous research evidences suggested that local and systemic inflammation play a pivotal role in the pathogenesis of ACS (Liuzzo, 2001; Elhajj et al., 2004). For example, the increase of IL-6, hs-CPR and CPR concentration can be observed in patients with systemic signs of systemic inflammation (Stefanadis et al., 2000; Centurión, 2016; Nanchen et al., 2019). In addition, FIB is the main determinant of thrombus formation, and its increase is related to the degree of coronary atherosclerosis, especially in patients with ACS (de Moerloose et al., 2010; Kurtul et al., 2016; Buljubasic et al., 2017).

RCTs were excluded if any of the following conditions were accord: (1) The therapy in RCTs includes PCI, or other TCM preparations. (2) The article data is wrong or incomplete. (3) The article could not be obtained full text. (4) Data duplicates. (5) No related outcome.

\section{Search Strategy}

Studies concerning RCTs published from inception to February 2020 were identified through a systematic electronic search of the following seven databases: the China National Knowledge Infrastructure Database (CNKI), the Chinese Scientific Journals Full-text Database (VIP), the Wan-Fang Database, the Chinese Biomedical Literature Database (SinoMed), the Cochrane Library, PubMed andEmbase. For this purpose, the free-text keywords and MESH (Medical Subject Heading) terms were lutilized, including "acute coronary syndrome [MeSH Terms]", "Acute Coronary Syndromes", "Coronary Syndrome, Acute", "Coronary Syndromes, Acute", "Syndrome, Acute Coronary", "Syndromes, Acute Coronary", "Danshen”, "Compound danshen", "Xiangdan”, "Fufangdanshen", "Danhong”, "Beitong”, "Salvianolate", "Salvianolic acid", "Danshenduofensuan”, "Danshenchuanxiongqin", "Sodium tanshinone IIA sulfonate", "Guanxinning" and "randomized controlled trial [Publication
Type]". In addition, there was no restrictions on blinding methods, publication year and language. Furthermore, the references of related articles were manually reviewed to identify further studies. The detailed and specific retrieval strategies are shown in Supplementary File 2.

\section{Data Extraction and Quality Assessment}

Data of eligible studies were independently extracted by two reviewers (SYG and $\mathrm{MWN}$ ) by using Microsoft Excel (Microsoft Corp, Redmond, WA). The specifically designed form captured information on the study characteristics, including publication data (publication date, title and authors' names), details of patients' characteristics (sample sizes, age and sex), intervention (the types of CHIs, dose and duration), outcomes (the primary and secondary outcomes) and factors to evaluate risk of bias.

According to the Cochrane risk of bias tool (Higgins et al., 2011), two investigators (SJ and JZ) independently conducted the quality assessment of all included RCTs. Each trial was scored as low, high, or unclear risk of bias on 7 quality evaluation items, including random sequence generation (selection bias), allocation concealment (selection bias), blinding of participants and personnel (performance bias), blinding of outcome assessment (detection bias), incomplete outcome data (attrition bias), selective reporting (reporting bias) and other bias. When discordance occurred in the process of literature selection, data extraction and quality assessment, the final results were resolved by adjudication with a third reviewer (WZ) or consensus.

\section{Statistical Analysis}

In consideration of the clinical and within-study and betweenstudy heterogeneity among the included RCTs, the randomeffect model was selected. Moreover, according to the Bayesian hierarchical model and Markov Chain Monte Carlo algorithm, WinBUGS 1.4.3 (MRC Biostatistics Unit, Cambridge, UK) software was adopted to analyze data. For dichotomous outcomes, the combined results were presented as odds ratios (ORs) with 95\% confidence intervals (95\% CIs). For continuous outcomes, the results were calculated as the mean differences (MDs) with 95\% CIs. If 95\% CIs of ORs did not include 1 and 95\% CIs of MDs did not contain 0, the differences between the groups would be considered statistically significant (Huang et al., 2019). In the WinBUGS program, we set 200,000 iterations, and the first 10000 iterations were regarded as burn-in for annealing to eliminate the influence of the initial value (Crainiceanu and Goldsmith, 2010).

Furthermore, the Stata V.13.0 software was utilized (Stata Corporation) to generate graphs in order to compare the relationship between different treatment measures. The surface under the cumulative ranking (SUCRA) area was employed to rank the probabilities for different interventions under each outcome. The SUCRA ranges from 0 to $100 \%$, assigning to the worst and best therapeutic measures, respectively (Cope and Jansen, 2013; Trinquart et al., 2016). In addition, the publication bias of included RCTs were checked though a comparison adjusted funnel plot (Chaimani et al., 2013). 


\section{Multidimensional Cluster Analysis}

The multidimensional cluster analysis was performed to assess comprehensive efficacy of competing treatments in any three outcomes based on SUCRA value. Hence, the "scatterplot3d" package in $\mathrm{R}$ 3.6.1 software (Mathsoft, Cambridge,USA) was used for multidimensional clustering analysis in this study. These interventions were clustered by using the k-means method, and the number of clusters was modified according to the actual situation (Ball and Hall, 1967; Haldar et al., 2008; Ahlqvist et al., 2018). The steps of clustering were as follows: (1) The included interventions were randomly divided into $\mathrm{k}$ initial categories, and the average of the outcome indicators of these $\mathrm{k}$ categories were regarded as the initial aggregation points. (2) An intervention was classified as the closest aggregation point category, and then the aggregation point of that category was updated to the mean of the present outcome indicators. All interventions were recategorized and classified and repeat step (2) until they have been assigned. Finally, the ranking of treatment interventions was visually displayed through a three-dimensional stereogram. The different colors dots in the figure represent different types of interventions.

\section{RESULTS}

\section{Literature Selection}

A total of 542 articles were obtained by searching 7 databases. After removing the duplicates, 183 articles need to be screened for the title and abstract. Then, 94 studies were excluded because of reviews, meta-analysis, system reviews, animal experiment and other irrelevant literature. Ultimately, 53 RCTs studies conducted in China from 2007 to 2019 were eligible in this NMA. The details of the articles screening process are depicted in Figure 1. Seven types of DSCIs were incorporated, including DS,

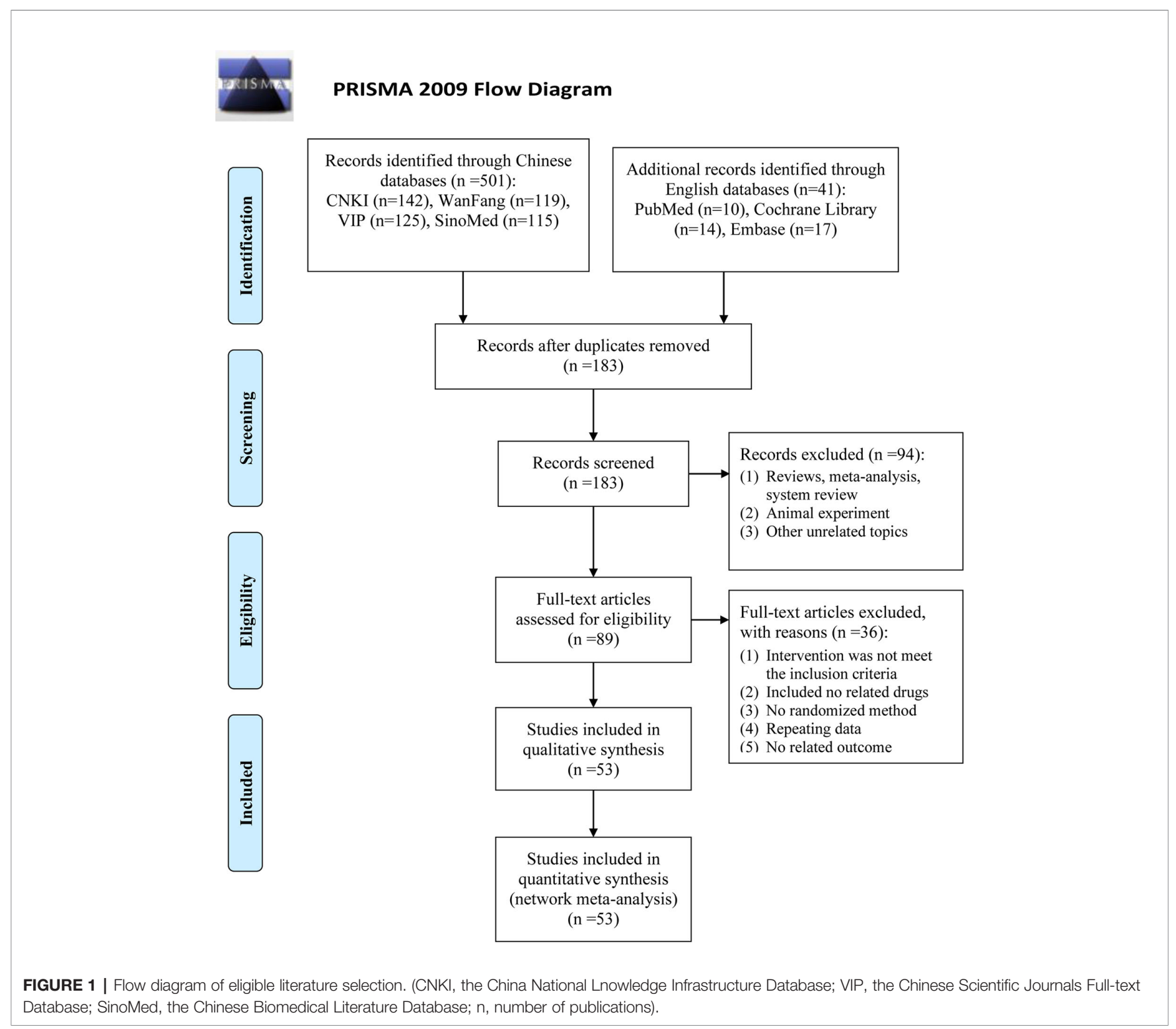


FFDS, DH, DSDFSY, DSCXQ, STS and GXN. The network graphs of the above $7 \mathrm{CHI}$ with different outcomes are described in Figure 2. The detailed information on CHIs shown in Supplementary File 3.

\section{Study Characteristics}

Overall, the 53 eligible RCTs studies involved 6,401 patients $(3,175$ in the control group, and 3,226 in the experiment group), and most of them were middle-aged and elderly. With the exception of five studies that did not report gender composition, male patients accounted for approximately $52.73 \%$ of the total study population. A total of nine comparisons were evaluated: DS + WM vs. WM ( $\mathrm{n}=$ 5), DSCXQ + WM vs. WM ( $\mathrm{n}=2)$, DSDFSY + WM vs. WM ( $\mathrm{n}=$ $5), \mathrm{DH}+\mathrm{WM}$ vs. $\mathrm{WM}(\mathrm{n}=30), \mathrm{FFDS}+\mathrm{WM}$ vs. WM $(\mathrm{n}=2)$, $\mathrm{GXN}+\mathrm{WM}$ vs. WM $(\mathrm{n}=4), \mathrm{DH}+\mathrm{WM}$ vs. FFDs + WM $(\mathrm{n}=3)$, $\mathrm{DH}+\mathrm{WM}$ vs. STS + WM $(\mathrm{n}=1)$, and $\mathrm{DH}+\mathrm{WM} / \mathrm{STS}+\mathrm{WM}$ vs.
FFDS + WM $(n=1)$. The therapies of the control group were $\mathrm{WM}$, primarily including nitrate, low molecular weight heparin, aspirin, nifedipine, statins, $\beta$-receptor blocker, etc. Moreover, the duration of treatment reported by most studies was 14 days. The details of the included study characteristics are shown in Table 1.

\section{Quality Evaluation}

In terms of random sequence generation (selection bias), a total of 6 RCTs used a random number table to generate random sequences, so they were considered to be low risk. Regarding performance bias, 1 RCT study mentioned the use of the single blind method, so it was evaluated as low risk. In terms of attrition bias, all studies had no incomplete data, so the evaluation was "low risk". Regarding reporting bias, 2 studies reported incomplete outcome indicators mentioned in the design plan, so they were regarded as high risk. In terms of other bias, five
A

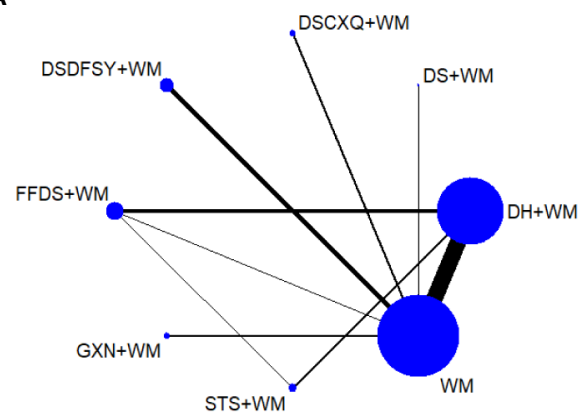

C

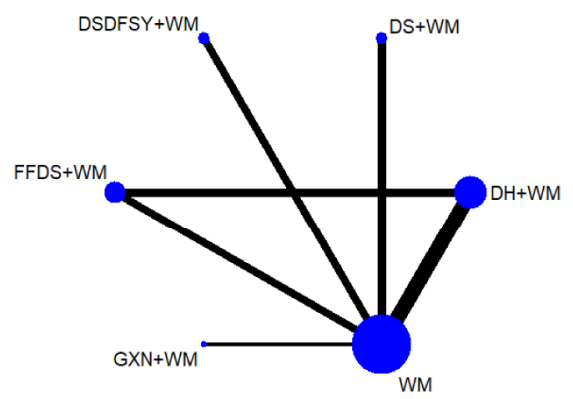

E

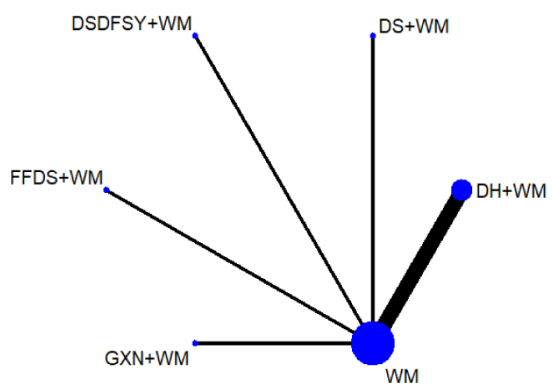

B

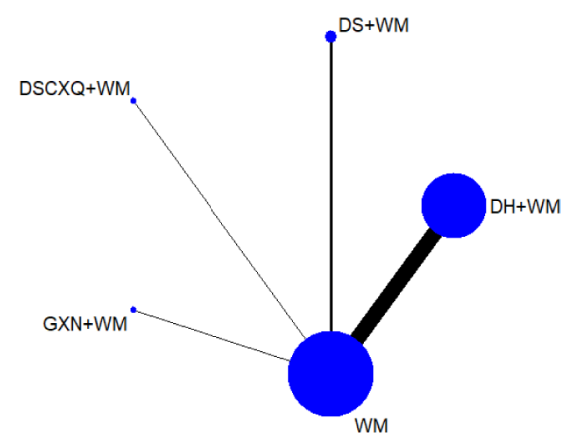

D

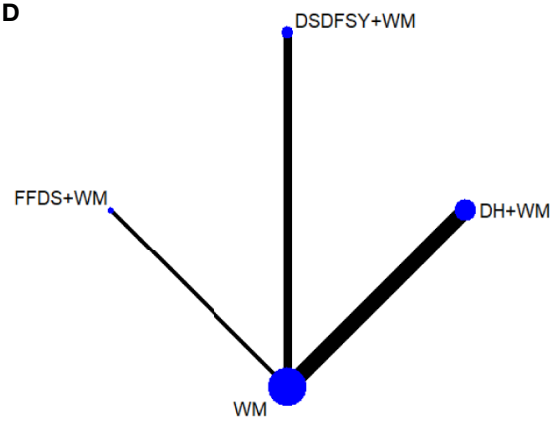

FIGURE 2 | Network graphs for different outcomes. (A) clinical effectiveness rate; (B) the level of hs-CRP; (C) the level of CRP; (D) the level of IL-6; (E) the level of FIB. (DS, Danshen injection; FFDS, Fufang Danshen injection; DH, Danhong injection; DSDFSY, Dansenduofensuanyan injection; DSCXQ, Danshenchuanxiongqin injection; STS, Sodium Tanshinone IIA Sulfonate injection; GXN, Guanxinning injection; WM, western medicine; hs-CRP, hypersensitive C-reactive protein; CRP, C-reactive protein; IL-6, interleukin-6; FIB, fibrinogen). 
TABLE 1 | Characteristics of the studies included in this AMA.

\begin{tabular}{|c|c|c|c|c|c|c|c|c|c|c|c|}
\hline \multirow[t]{2}{*}{ Study ID } & \multicolumn{2}{|c|}{ Sample size } & \multicolumn{2}{|c|}{$\operatorname{Sex}(M / F)$} & \multicolumn{2}{|c|}{ Average age } & \multirow[t]{2}{*}{ Therapy of experiment group } & \multirow[t]{2}{*}{ Menstruum } & \multirow[t]{2}{*}{$\begin{array}{l}\text { Therapy of } \\
\text { control group }\end{array}$} & \multirow[t]{2}{*}{ Course (days) } & \multirow[t]{2}{*}{ Outcomes } \\
\hline & E & C & E & C & E & C & & & & & \\
\hline Li et al., 2007 & 37 & 36 & \multicolumn{2}{|c|}{$39 / 34$} & \multicolumn{2}{|c|}{$67.4 \pm 6.5$} & DS20ml+WM & NR & WM & $14 d$ & (2) \\
\hline Shi, 2013 & 30 & 30 & \multicolumn{2}{|c|}{$34 / 26$} & \multicolumn{2}{|c|}{$63.5 \pm 7.4$} & DS20ml+WM & $0.9 \% N S 480 \mathrm{~mL}$ & WM & NR & (1) 5 \\
\hline Long, 2011 & 35 & 35 & \multicolumn{2}{|c|}{$35 / 35$} & \multicolumn{2}{|c|}{67} & DS+WM & NR & WM & $14 d$ & (2) \\
\hline Ma and Liang, 2016 & 60 & 60 & $30 / 30$ & $32 / 28$ & $72.22 \pm 7.18$ & $71.88 \pm 7.04$ & DS20ml+WM & $5 \%$ GS $250 \mathrm{~mL}$ & WM & $14 d$ & (3) \\
\hline Su and Zhao, 2017 & 59 & 59 & $33 / 26$ & $31 / 28$ & $69.46 \pm 6.67$ & $69.44 \pm 6.65$ & DS20ml+WM & NR & WM & $14 d$ & (3) \\
\hline Wu et al., 2009 & 50 & 48 & $28 / 22$ & $27 / 21$ & 54 79 & 52 80 & DSCXQ10ml+WM & $5 \% \mathrm{GS} / \mathrm{NS} 500 \mathrm{~mL}$ & WM & $10 d$ & (1)(2) \\
\hline Zhang et al., 2014 & 713 & 800 & $371 / 342$ & $433 / 367$ & $43 \sim 77$ & 39 74 & DSCXQ10ml+WM & NR & WM & $14 d$ & (1)(6) \\
\hline Lin and Yu, 2017 & 45 & 44 & $27 / 18$ & $24 / 20$ & $67.1 \pm 6.7$ & $66.9 \pm 6.1$ & DSDFSY200mg+WM & $5 \%$ GS $250 \mathrm{~mL}$ & WM & $14 d$ & (1)6 \\
\hline Xia et al., 2017 & 31 & 35 & $20 / 11$ & $22 / 13$ & $61.5 \pm 11.4$ & $62.1 \pm 12.6$ & DSDFSY200mg+WM & $0.9 \% \mathrm{NS} 250 \mathrm{~mL}$ & WM & $14 d$ & (1)(4) \\
\hline Li et al., 2019 & 54 & 54 & $29 / 25$ & $30 / 24$ & $60.17 \pm 5.14$ & $60.01 \pm 5.28$ & DSDFSY200mg+WM & $5 \%$ GS $250 \mathrm{~mL}$ & WM & $1 \mathrm{~m}$ & (1)(3) 4 \\
\hline Li, 2013 & 24 & 22 & $14 / 10$ & $12 / 10$ & $66 \pm 8$ & $60 \pm 5$ & DSDFSY200mg+WM & 5\%GS 250mL & WM & $14 d$ & (1)356 \\
\hline Lin, 2015 & 30 & 30 & \multicolumn{2}{|c|}{$39 / 21$} & \multicolumn{2}{|c|}{$67.20 \pm 7.18$} & DSDFSY200mg+WM & $10 \%$ GS $250 \mathrm{~mL}$ & WM & $14 d$ & (1) \\
\hline $\begin{array}{l}\text { Wang and Zhang, } \\
2012\end{array}$ & 43 & 43 & $28 / 15$ & $29 / 14$ & 76 91 & 76 88 & $\mathrm{DH} 2 \mathrm{Oml}+\mathrm{WM}$ & $\begin{array}{l}5 \% \mathrm{GS} / 0.9 \% \mathrm{NS} \\
250 \mathrm{~mL}\end{array}$ & WM & $14 d$ & (1)(3) \\
\hline Xu, 2011 & 42 & 40 & $25 / 17$ & $22 / 18$ & $42-78$ & $41-76$ & DH4Oml+WM & 5\%GS 250mL & WM & $14 d$ & (1) \\
\hline Li, 2015 & 90 & 90 & $60 / 30$ & $58 / 32$ & $67.8 \pm 3.2$ & $68.5 \pm 4.1$ & DH2Oml+WM & $\begin{array}{l}5 \% \mathrm{GS} / 0.9 \% \mathrm{NS} \\
250 \mathrm{~mL}\end{array}$ & WM & $14 d$ & (1)(3) \\
\hline Liu et al., 2008 & 150 & 150 & $101 / 49$ & $98 / 52$ & $57.1 \pm 6.2$ & $55.6 \pm 7.1$ & $\mathrm{DH} 40 \mathrm{ml}+\mathrm{WM}$ & $\begin{array}{l}5 \% \mathrm{GS} / 0.9 \% \mathrm{NS} \\
250 \mathrm{~mL}\end{array}$ & WM & $14 d$ & (1) 6 \\
\hline Ding, 2012 & 30 & 30 & $13 / 17$ & $12 / 18$ & $66.50 \pm 7.10$ & $65.90 \pm 7.30$ & $\mathrm{DH} 40 \mathrm{ml}+\mathrm{WM}$ & $0.9 \% \mathrm{NS} 250 \mathrm{~mL}$ & WM & $14 d$ & (1) \\
\hline Yang, 2010 & 40 & 40 & \multicolumn{2}{|c|}{$43 / 37$} & \multicolumn{2}{|c|}{$58.85 \pm 6.8$} & DH2Oml+WM & NS $250 \mathrm{~mL}$ & WM & $14 d$ & (1) \\
\hline Du and Chen, 2009 & 45 & 44 & \multicolumn{2}{|c|}{$54 / 35$} & \multicolumn{2}{|c|}{$68.4 \pm 7.2$} & DH2Oml+WM & $5 \%$ GS $250 \mathrm{~mL}$ & WM & $14 d$ & (3) \\
\hline Zhou, 2011 & 30 & 30 & & & $N$ & & $\mathrm{DH} 20 \mathrm{mg}+\mathrm{WM}$ & $5 \%$ GS/NS 250mL & WM & $14 d$ & (1)(26) \\
\hline Nie and Zhao, 2012 & 30 & 30 & & & 42 & & $\mathrm{DH} 40 \mathrm{ml}+\mathrm{WM}$ & $5 \%$ GS/NS $250 \mathrm{~mL}$ & WM & $15 d$ & (2) \\
\hline Su and Jiang, 2012 & 43 & 43 & & & $63.2=$ & 0.7 & $\mathrm{DH} 4 \mathrm{Oml}+\mathrm{WM}$ & NS $250 \mathrm{~mL}$ & WM & $14 d$ & (2) \\
\hline Gu et al., 2011 & 70 & 70 & & & $N$ & & $\mathrm{DH} 4 \mathrm{Oml}+\mathrm{WM}$ & 5\%GS 250mL & WM & $14 d$ & (3) \\
\hline Liang et al., 2019 & 35 & 35 & $21 / 14$ & 22/13 & $62.8 \pm 5.3$ & $63.1 \pm 5.5$ & DH4Oml+WM & $0.9 \% N S 250 \mathrm{~mL}$ & WM & $14 d^{*} 2$ & (2)(4) \\
\hline Cao et al., 2010 & 47 & 35 & $30 / 17$ & $21 / 14$ & $71.5 \pm 4.5$ & $69.9 \pm 7.2$ & $\mathrm{DH} 40 \mathrm{ml}+\mathrm{WM}$ & $5 \% G S$ & WM & $14 d$ & (5) \\
\hline Feng, 2011 & 30 & 30 & & & $\mathrm{~N}$ & & DH2Omg+WM & $5 \% \mathrm{GS} / \mathrm{NS} 250 \mathrm{~mL}$ & WM & $14 \mathrm{~d}$ & (1)(20) \\
\hline Wei, 2012 & 61 & 61 & & & 59.4 & & DH2Oml+WM & NR & WM & $10 \mathrm{~d}$ & (1)(2) \\
\hline Guan et al., 2010 & 60 & 60 & & & $73.54 \pm 10.12$ & $72.15 \pm 10.03$ & DH3Oml+WM & NS 250 mL & WM & $14 d$ & (2)(4) \\
\hline Zhang et al., 2010 & 65 & 60 & $34 / 31$ & $32 / 28$ & $65.4 \pm 4.6$ & $65.8 \pm 4.3$ & DH3Oml+WM & 5\%GS/NS 250mL & WM & $28 d$ & (24)(6) \\
\hline Chen, 2012 & 30 & 30 & 31 & & 50 & & $\mathrm{DH} 40 \mathrm{ml}+\mathrm{WM}$ & $5 \%$ GS/NS 250mL & WM & $14 d$ & (1) 6 \\
\hline Chen and Wang, 2011 & 45 & 45 & & & $67 \pm$ & & DH4Oml+WM & $5 \%$ GS/NS 250mL & WM & $14 d$ & (1) 6 \\
\hline Li et al., 2010 & 42 & 38 & & & 58.3 & & $\mathrm{DH} 30 \mathrm{ml}+\mathrm{WM}$ & $5 \%$ GS/NS $250 \mathrm{~mL}$ & WM & $14 d$ & (2) \\
\hline Li et al., 2007 & 20 & 20 & & & $M: 66 \pm 9.5$ & $F: 65 \pm 6.6$ & DH2OmL+WM & NR & WM & $14 d$ & (1) \\
\hline Li and Song, 2011 & 34 & 32 & 22/10 & $18 / 11$ & $60.12 \pm 4.51$ & $58.46 \pm 6.25$ & DH20 30mL+WM & NS 250 mL & WM & $14 d$ & (2) \\
\hline Du, 2011 & 30 & 30 & & & $N$ & & DH2Omg+WM & $5 \%$ GS/NS 250mL & WM & $14 d$ & (1)6 \\
\hline Li and Meng, 2008 & 26 & 22 & $16 / 10$ & $13 / 9$ & 56 82 & $49 \pm 76$ & $\mathrm{DH} 40 \mathrm{ml}+\mathrm{WM}$ & $5 \%$ GS/NS 500mL & WM & $14 d$ & (1)6 \\
\hline Wang et al., 2015 & 123 & 122 & $82 / 41$ & $80 / 42$ & $83.4 \pm 6.4$ & $83.2 \pm 7.2$ & $\mathrm{DH} 40 \mathrm{ml}+\mathrm{WM}$ & $5 \%$ GS/NS 250mL & WM & $14 d$ & (1)6 \\
\hline Lao et al., 2013 & 35 & 35 & $19 / 16$ & $18 / 17$ & $62.27 \pm 2.29$ & $63.58 \pm 2.25$ & DH2OmL+WM & $5 \% G S / N S$ 250mL & WM & $56 d$ & (2)6 \\
\hline Fei et al., 2010 & 60 & 60 & & & $73.54 \pm 10.12$ & $72.15 \pm 10.03$ & $\mathrm{DH} 30 \mathrm{~mL}+\mathrm{WM}$ & NS 250 mL & WM & $14 d$ & (4) \\
\hline Zhang et al., 2012 & 44 & 44 & $22 / 22$ & $26 / 18$ & 22 41 & 20 44 & $\mathrm{DH} 20 \mathrm{~mL}+\mathrm{WM}$ & 10\%NS 250 mL & WM & $10 d$ & (1)6 \\
\hline $\mathrm{He}, 2014$ & 36 & 36 & $22 / 14$ & 20/16 & 56. $8 \pm 9.4$ & $57.1 \pm 9.8$ & $\mathrm{DH}+\mathrm{WM}$ & $5 \% \mathrm{GS} / \mathrm{NS} 250 \mathrm{~mL}$ & WM & $14 d$ & (1) \\
\hline Li and Ge, 2011 & 44 & 42 & & & 64.5 & & $\mathrm{DH} 2 \mathrm{OmL}+\mathrm{WM}$ & $\begin{array}{l}10 \% \mathrm{GS} / \mathrm{NS} \\
250 \mathrm{~mL}\end{array}$ & WM & $10 d$ & (1)(26) \\
\hline Hong et al., 2004 & 90 & 30 & $52 / 38$ & $17 / 13$ & $62.95 \pm 9.36$ & $66.00 \pm 8.96$ & FFDS20ml+WM & $5 \%$ GS/NS $250 \mathrm{~mL}$ & WM & $14 d$ & (3)(4) \\
\hline
\end{tabular}




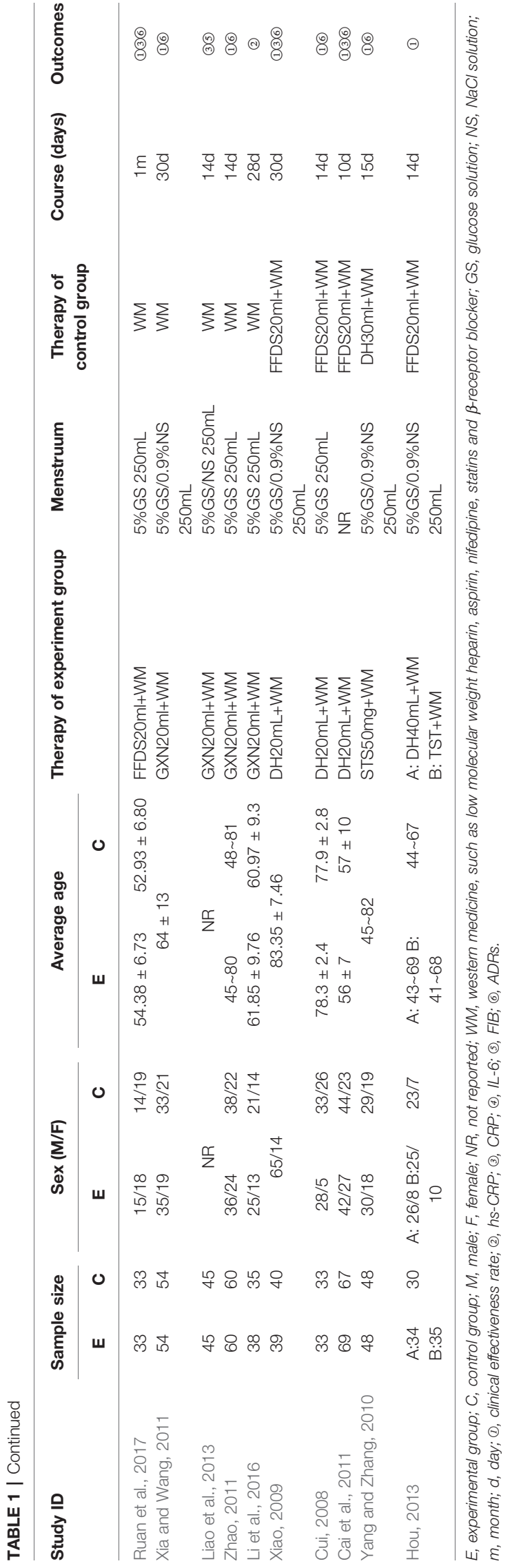

studies did not report whether the experiment group and the control group were comparable at baseline, which might affect the study results, so they were rated as high risk. In addition, the risk bias entries of the remaining studies were considered "unclear" due to insufficient information. In general, the quality of included studies was not high. The risk of bias for each eligible RCTs is depicted in Figure 3.

\section{Outcomes}

\section{Clinical Effectiveness Rate}

A total of 34 RCTs referred to the clinical effectiveness rate (DS + $\mathrm{WM}$ vs. WM $(\mathrm{n}=1), \mathrm{DSCXQ}+\mathrm{WM}$ vs. WM $(\mathrm{n}=2), \mathrm{DSDFSY}+$ $\mathrm{WM}$ vs. $\mathrm{WM}(\mathrm{n}=5), \mathrm{DH}+\mathrm{WM}$ vs. $\mathrm{WM}(\mathrm{n}=18), \mathrm{FFDS}+\mathrm{WM}$ vs. $\mathrm{WM}(\mathrm{n}=1), \mathrm{GXN}+\mathrm{WM}$ vs. $\mathrm{WM}(\mathrm{n}=2), \mathrm{DH}+\mathrm{WM}$ vs. FFDs + $\mathrm{WM}(\mathrm{n}=3), \mathrm{DH}+\mathrm{WM}$ vs. STS + WM $(\mathrm{n}=1)$, and $\mathrm{DH}+\mathrm{WM} /$ STS + WM vs. FFDS + WM $(n=1))$, including 7 types of CHIs. Apart from DS and FFDS injection, other CHIs combined with WM were superior to WM alone, and the difference was statistically significant. Table 2 shows the following specific outcomes: DH + WM vs. WM (OR= 0.21, 95\% CIs: 0.15-0.29), DSDFSY + WM vs. $\mathrm{WM}(\mathrm{OR}=0.24,95 \%$ CIs: $0.12-0.45)$, DSCXQ + WM vs. WM (OR= $0.57,95 \%$ CIs: $0.27-0.90)$, STS + WM vs. WM (OR $=0.31,95 \%$ CIs: $0.12-0.82)$ and $\mathrm{GXN}+\mathrm{WM}$ vs. WM (OR= $0.32,95 \%$ CIs: $0.13-$ 0.76). In addition, compared with the efficacy of FFDS + WM, DH + $\mathrm{WM}(\mathrm{OR}=2.96,95 \%$ CIs: 1.71-5.20) and DSDFSY + WM $(\mathrm{OR}=$ 2.63, 95\% CIs: 1.07-6.49) were related to significantly improved clinical outcomes. There was no statistically significant difference between other interventions.

According to the ranking of SUCRA probabilities (Figure 4, Table 3), DH + WM (81.8\%) was the most likely to become the best intervention for improving the clinical effectiveness rate. Moreover, the other 6 CHIs were ranked as follows: DSDFSY+WM (73.4\%) > DS+WM $(72.1 \%)>$ STS+WM $(59.3 \%)>$ GXN+WM $(58.3 \%)>$ DSCXQ+WM (29.9\%) > FFDS+WM (23.4\%).

\section{The Level of hs-CRP}

A total of 16 RCTs involving 4 interventions investigated hs-CRP [DS + WM vs. WM $(\mathrm{n}=2), \mathrm{DSCXQ}+\mathrm{WM}$ vs. WM $(\mathrm{n}=1), \mathrm{DH}+$ $\mathrm{WM}$ vs. WM $(\mathrm{n}=12)$ and GXN + WM vs. WM $(\mathrm{n}=1)]$. The specific outcomes are described in Table 2. $\mathrm{DH}+\mathrm{WM}(\mathrm{MD}=-1.43$, 95\% CIs: $-3.24 \sim-0.25$ ) could achieve a better effect in hs-CRP than WM alone, and the difference was statistically significant. Simultaneously, there was no statistically significant difference between other interventions. Base on the data of SUCRA probabilities (Figure 4, Table 3), the 4 CHIs were ranked as follows: DSCXQ+WM $(76.1 \%)>$ DS+WM $(60.5 \%)>$ DH+WM (54.1\%) > GXN+WM (39.4\%).

\section{The Level of CRP}

In total, 13 RCTs with 5 CHIs observed data regarding CRP [DS + $\mathrm{WM}$ vs. WM $(\mathrm{n}=2), \mathrm{DSCXQ}+\mathrm{WM}$ vs. $\mathrm{WM}(\mathrm{n}=2), \mathrm{DH}+\mathrm{WM}$ vs. $\mathrm{WM}(\mathrm{n}=4), \operatorname{FFDS}+\mathrm{WM}(\mathrm{n}=2), \mathrm{GXN}+\mathrm{WM}(\mathrm{n}=1)$ and $\mathrm{DH}+$ $\mathrm{WM}$ vs. FFDs + WM $(\mathrm{n}=2)]$. The results demonstrated that no significant differences, which are shown in Table 2. According to the SUCRA values (Figure 4, Table 3), the 5 CHIs were ranked as follows: $\mathrm{DH}+\mathrm{WM}(75.3 \%)>\mathrm{DS}+\mathrm{WM}(63.7 \%)>\mathrm{FFDS}+\mathrm{WM}$ $(55 \%)>$ GXN+WM $(53.7 \%)>$ DSDFSY+WM (40.7\%). 


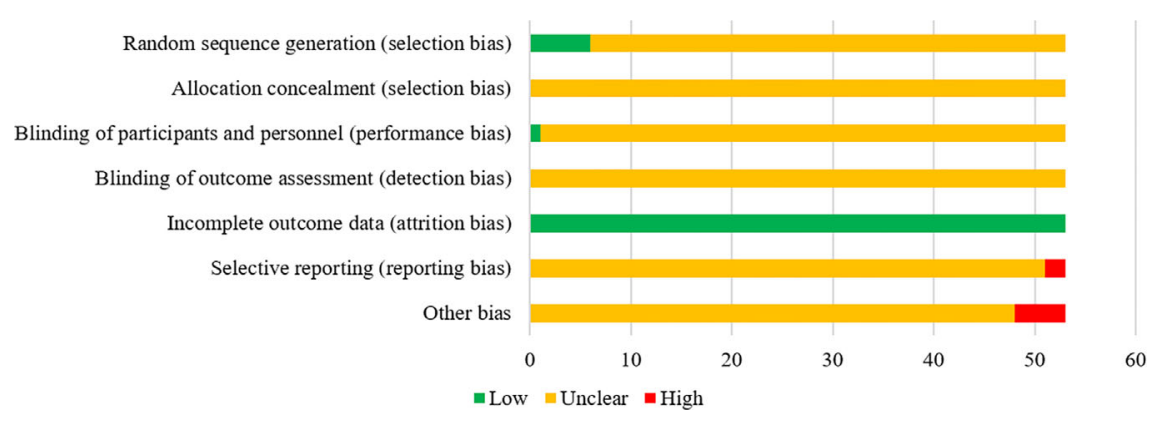

FIGURE 3 | The risk of bias of the included RCTs. The vertical axis represents the quality evaluation items and horizontal axis represents the number of RCTs.

TABLE 2 | Odds ratio/mean difference (95\%Cls) of all therapeutic measures.

\begin{tabular}{|c|c|c|c|c|c|}
\hline Comparison & Clinical effectiveness rate & hs-CRP & CRP & IL-6 & FIB \\
\hline \multicolumn{6}{|l|}{ DS+WM vs } \\
\hline FFDS+WM & $0.34(0.03,1.99)$ & - & $-0.34(-6.01,4.66)$ & - & $-0.56(-6.23,5.26)$ \\
\hline $\mathrm{DH}+\mathrm{WM}$ & $1.02(0.11,5.50)$ & $-1.56(-11.00,9.00)$ & $0.33(-4.21,5.02)$ & - & $-0.56(-5.02,3.98)$ \\
\hline DSDFSY+WM & $0.90(0.09,5.23)$ & - & $-1.10(-5.84,3.53)$ & - & $-0.76(-6.04,4.65)$ \\
\hline DSCXQ+WM & $0.39(0.04,2.27)$ & $1.37(-10.92,13.58)$ & - & - & - \\
\hline STS+WM & $0.68(0.06,4.65)$ & - & - & - & - \\
\hline GXN+WM & $0.67(0.07,4.48)$ & $-2.29(-13.59,9.53)$ & $-0.52(-5.72,4.59)$ & - & $-0.37(-5.72,5.07)$ \\
\hline WM & $0.21(0.02,1.12)$ & $-3.08(-12.48,7.28)$ & $-2.18(-5.85,1.39)$ & - & $-0.99(-5.03,3.18)$ \\
\hline \multicolumn{6}{|l|}{ FFDS+WM vs } \\
\hline $\mathrm{DH}+\mathrm{WM}$ & $2.96(1.71,5.20)$ & - & $0.60(-1.98,4.57)$ & $-2.63(-51.87,46.86)$ & $0.01(-4.48,4.37)$ \\
\hline DSDFSY+WM & $2.63(1.07,6.49)$ & - & $-0.76(-5.39,4.50)$ & $-2.77(-48.31,42.31)$ & $-0.18(-5.53,5.11)$ \\
\hline DSCXQ+WM & $1.12(0.52,2.87)$ & - & - & - & - \\
\hline STS+WM & $2.02(0.77,5.27)$ & - & - & - & - \\
\hline GXN+WM & $1.97(0.68,6.03)$ & - & $-0.20(-5.23,5.65)$ & - & $0.17(-5.18,5.53)$ \\
\hline WM & $0.62(0.33,1.17)$ & - & $-1.87(-5.42,2.42)$ & $-4.93(-49.18,38.96)$ & $-0.44(-4.49,3.58)$ \\
\hline \multicolumn{6}{|l|}{$\mathrm{DH}+\mathrm{WM}$ vs } \\
\hline DSDFSY+WM & $0.88(0.43,1.86)$ & - & $-1.41(-5.75,2.49)$ & $-0.10(-23.09,22.02)$ & $-0.21(-4.06,3.79)$ \\
\hline DSCXQ+WM & $0.38(0.21,0.81)$ & $2.85(-4.43,9.85)$ & - & - & - \\
\hline STS+WM & $0.68(0.27,1.70)$ & - & - & - & - \\
\hline GXN+WM & $0.67(0.25,1.78)$ & $-0.68(-6.89,5.30)$ & $-0.83(-5.74,3.66)$ & - & $0.18(-3.74,4.22)$ \\
\hline WM & $0.21(0.15,0.29)$ & $-1.43(-\underline{3.24,},-0.25)$ & $-2.51(-5.47,0.13)$ & - & $-0.45(-2.24,1.42)$ \\
\hline \multicolumn{6}{|c|}{ DSDFSY+WM vs } \\
\hline DSCXQ+WM & $0.43(0.19,1.09)$ & - & - & - & - \\
\hline STS+WM & $0.77(0.24,2.41)$ & - & - & - & - \\
\hline GXN+WM & $0.76(0.25,2.30)$ & - & $0.56(-4.18,5.45)$ & - & $0.39(-4.57,5.35)$ \\
\hline WM & $0.24(0.12,0.45)$ & - & $-1.11(-4.05,1.94)$ & $-2.12(-11.43,7.12)$ & $-0.22(-3.73,3.19)$ \\
\hline \multicolumn{6}{|l|}{ DSCXQ+WM vs } \\
\hline STS+WM & $1.79(0.54,5.15)$ & - & - & - & - \\
\hline GXN+WM & $1.75(0.57,4.89)$ & $-3.59(-12.64,5.75)$ & - & - & - \\
\hline WM & $0.57(0.27,0.90)$ & $-4.36(-11.26,2.76)$ & - & - & - \\
\hline \multicolumn{6}{|l|}{ STS+WM vs } \\
\hline GXN+WM & $0.98(0.27,3.72)$ & - & - & - & - \\
\hline WM & $\underline{0.31(0.12,0.82)}$ & - & - & - & - \\
\hline \multicolumn{6}{|l|}{ GXN+WM vs } \\
\hline WM & $0.32(0.13,0.76)$ & $-0.80(-6.70,5.13)$ & $-1.68(-5.48,2.14)$ & - & $-0.62(-4.18,2.91)$ \\
\hline
\end{tabular}

The bolded and underlined results indicate statistical significance.

\section{The Level of IL-6}

A total of 7 RCTs with 3 CHIs presented data about IL-6 [DSDFSY + $\mathrm{WM}$ vs. WM $(\mathrm{n}=2), \mathrm{DH}+\mathrm{WM}$ vs. WM $(\mathrm{n}=4)$ and FFDS+WM $(n=1)]$. Additionally, the results demonstrated that no significant differences in IL- 6 was observed among the above CHIs, which are shown in Table 2. In terms of the SUCRA values (Figure 4, Table 3), the 3 CHIs were ranked as follows: FFDS+WM (56.2\%) > DSDFSY+WM (54\%) > DH+WM (51.6\%).

\section{The Level of FIB}

The data on the FIB were available for 8 RCTs including 5 types of CHIs [DS + WM vs. WM ( $n=1)$, DSDFSY + WM vs. WM $(n=1)$, 

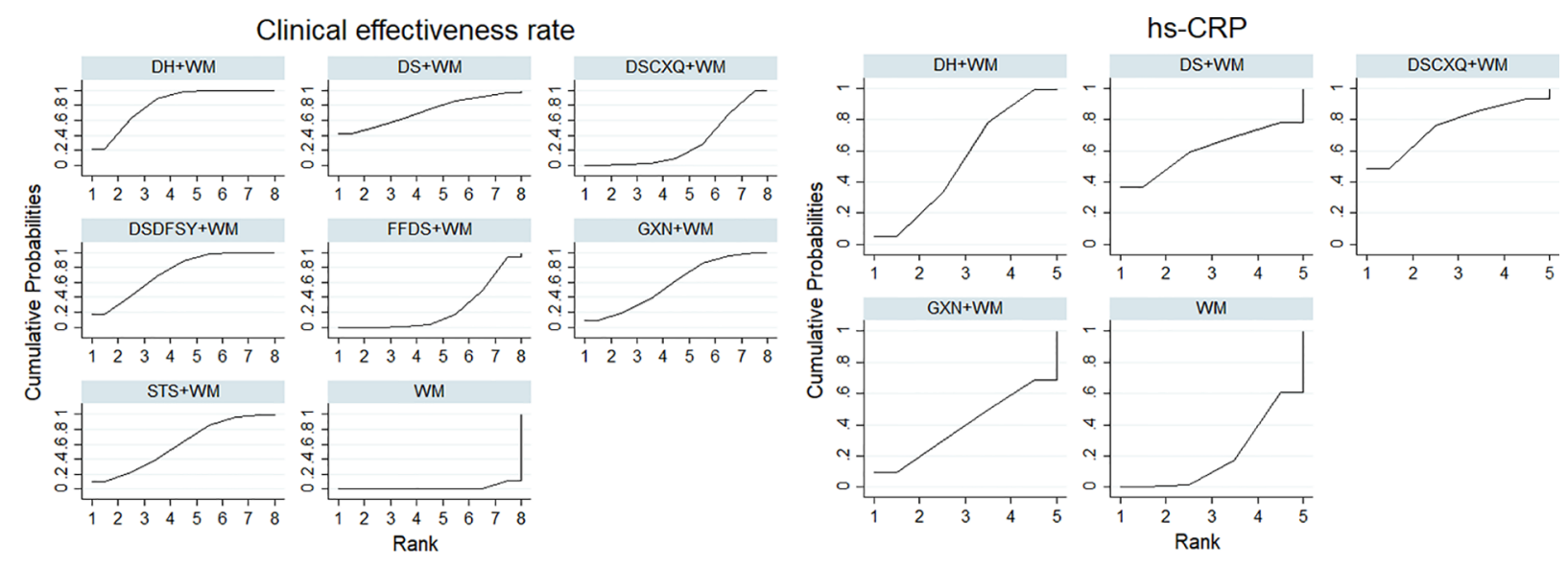

CRP
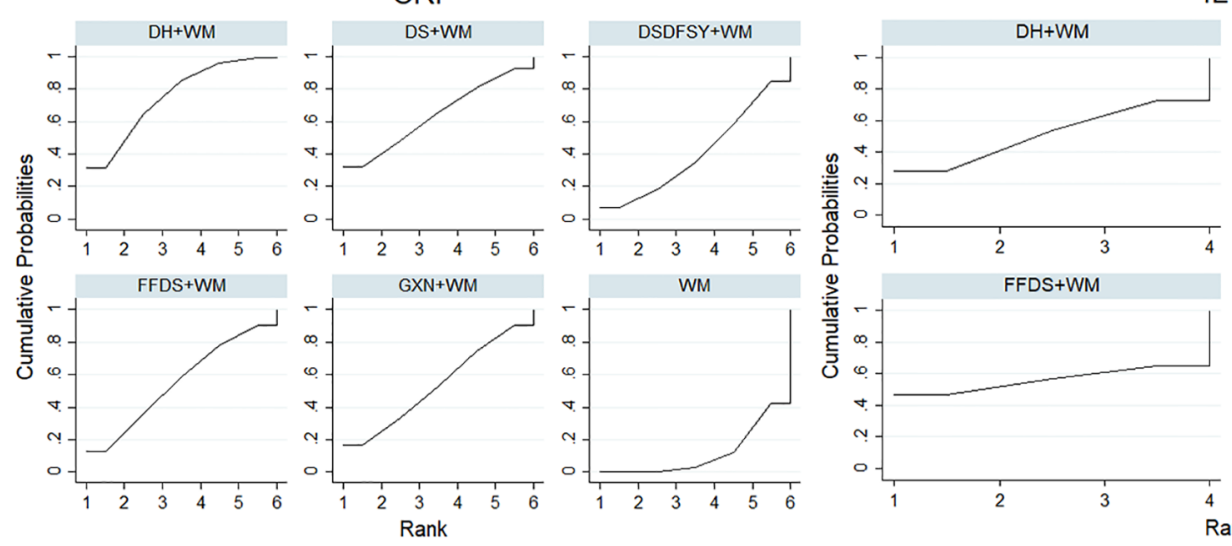

IL-6

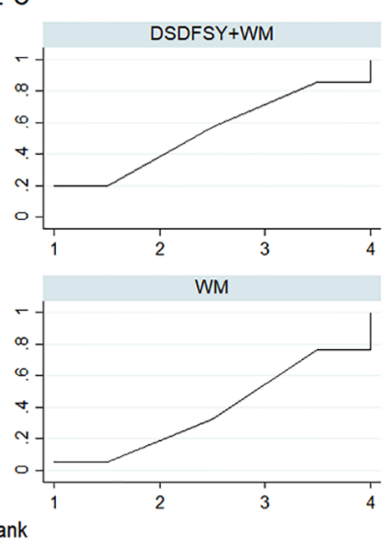

FIB
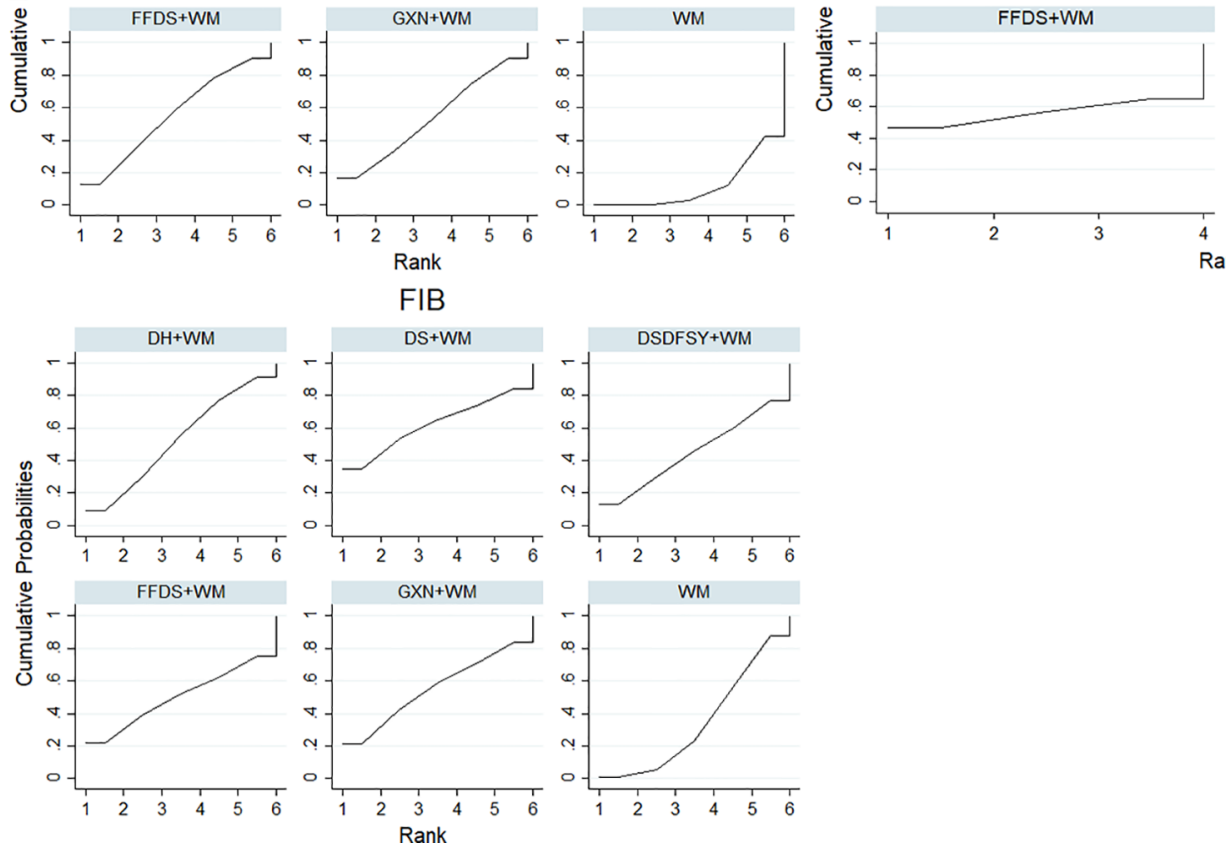

FIGURE 4 | SUCRA for different outcomes. The vertical axis represents cumulative probabilities and horizontal axis represents rank. (DS, Danshen injection; FFDS, Fufang Danshen injection; DH, Danhong injection; DSDFSY, Dansenduofensuanyan injection; DSCXQ, Danshenchuanxiongqin injection; STS, Sodium Tanshinone IIA Sulfonate injection; GXN, Guanxinning injection; WM, western medicine; hs-CRP, hypersensitive C-reactive protein; CRP, C-reactive protein; IL-6, interleukin-6; FIB, fibrinogen).

$\mathrm{DH}+\mathrm{WM}$ vs. WM $(\mathrm{n}=4), \mathrm{GXN}+\mathrm{WM}$ vs. WM $(\mathrm{n}=1)$ and FFDS+WM $(n=1)]$. Simultaneously, the results showed that no significant differences among above CHIs groups for FIB (Table 2). According to the SUCRA values (Figure 4, Table 3), the 5 CHIs were ranked as follows: DS+WM (62\%) > GXN+WM (55.4\%) > DH+WM $(52.5 \%)>\operatorname{FFDS}+\mathrm{WM}(50.2 \%)>$ DSDFSY+WM $(45.2 \%)$.

\section{Safety}

In terms of safety, 28 studies did not address ADRs, and 17 studies indicated that there were no significant ADRs. Meanwhile, 1 study (Li Y M, 2007) only reported monitoring ADRs related to liver and kidney dysfunction and bleeding tendency, but did not report the corresponding results. In 
TABLE 3 | SUCRA (\%) of all therapeutic measures.

\begin{tabular}{|c|c|c|c|c|c|}
\hline Intervention & Clinical effectiveness rate & hs-CRP & CRP & IL-6 & FIB \\
\hline DS+WM & 72.1 & 60.5 & 63.7 & - & 62 \\
\hline FFDS+WM & 23.4 & - & 55 & 56.2 & 50.2 \\
\hline $\mathrm{DH}+\mathrm{WM}$ & 81.8 & 54.1 & 75.3 & 51.6 & 52.5 \\
\hline DSDFSY+WM & 73.4 & - & 40.7 & 54 & 45.2 \\
\hline$D S C X Q+W M$ & 29.9 & 76.1 & - & - & - \\
\hline STS+WM & 59.3 & - & - & - & - \\
\hline GXN+WM & 58.3 & 39.4 & 53.7 & - & 55.4 \\
\hline WM & 1.8 & 19.9 & 11.7 & 38.2 & 34.7 \\
\hline
\end{tabular}

The redder color in the table means the higher the ranking of the corresponding interventions.

addition, 1 study (Yang and Zhang, 2010) only described 3 cases of ADRs in the experiment group (STS+WM) and 2 cases in the control group $(\mathrm{DH}+\mathrm{WM})$, but none of them affected the treatment. In Yang's research (Lin and Yu, 2017), 3 cases (allergic rash, vomiting and bloating) and 2 cases (vomiting) of ADRs occurred in the experiment group (DSDFSY+WM) and the control group (WM), respectively, and the symptoms were mild. In Lao's study (Lao et al., 2013), one patient in the control group (WM) had elevated transaminase and returned to normal levels after hepatoprotective treatment; none of the experiment group ( $\mathrm{DH}+\mathrm{WM}$ ) had abnormal liver and kidney test indicators during the treatment process, and there were no serious ADRs. In Ruan's study (Ruan et al., 2017), 4 ADRs occurred in the experiment group (FFDS+WM), including drowsiness, fever and mild gastrointestinal reactions; 5 ADRs occurred in the control group (WM), containing dizziness, drowsiness and mild gastrointestinal reactions. In the research of Xia and Whang (2011), 1 patient in the experiment group (GXN+WM) presented with facial redness and mild headache, and 2 had rashes.; the control group (WM) developed 2 cases of flushing and mild headache, 1 case of rash, and 1 case of local swelling by injection. In the study Zhao (2011), some patients in the experiment group $(\mathrm{GXN}+\mathrm{WM})$ and the control group (WM) developed headaches, which were relieved by reducing nitrate dosage. In the research of Xiao (2009), 1 patient in the experimental group $(\mathrm{DH}+\mathrm{WM})$ developed facial redness and mild headache, while 2 patients in the control group (FFDS +WM) developed a sense of local swelling from the injection. However, since most eligible RCTs studies did not focus on the monitoring of ADRs, the safety of these CHIs needs to be further explored.

\section{Multidimensional Cluster Analysis}

When cluster analysis was conducted to 4 interventions that reported the hs-CRP, clinical effectiveness rate and CRP, DH +WM was superior to the other regimens, and WM only was evaluated the worst (Figure 5A). In terms of the clinical effectiveness rate, hs-CRP and FIB, DS + WM and DH + WM were similarly superior (Figure 5B). Furthermore, Regarding the clinical effectiveness rate, CRP and IL-6, DH+WM was similarly preferred (Figure 5C). Moreover, DS + WM and DH + WM were dominant in the comprehensive ranking of the clinical effectiveness rate, CRP and FIB (Figure 5D). With regard to the comprehensive ranking of the clinical effectiveness rate, FIB and
IL-6, the results indicated that $\mathrm{DH}+\mathrm{WM}$ had the potential to be the best intervention (Figure 5E). In summary, the results of the multidimensional cluster analysis demonstrated that $\mathrm{DH}+\mathrm{WM}$ and DS+WM might have better therapeutic effects.

\section{Publication Bias}

This study drew a Comparison-adjusted funnel plot for clinical effectiveness rate to test publication bias. When the distribution points in the funnel chart are symmetrical, it means that there is no publication bias (Riley et al., 2011). As depicted in Figure 6, the points in the funnel chart were asymmetric based on the position of the center line, and the angle between the adjusted auxiliary line and the center line was large, indicating a potential publication bias in this study.

\section{Consistency Test}

As depicted in Figure 7, there were 2 three-side rings. The results indicated that the 95\% CIs contained 0, and IF was between 0.66 and 1.23. Consequently, there was some inconsistency in this NMA study.

\section{DISCUSSION}

We performed the approach of current NMA to evaluate the comparative efficacy and safety of reported DSCIs combination with WM in the treatment of ACS. This study included 53 eligible RCTs with 7 CHIs that evaluated the clinical effectiveness rate, the level of hs-CRP, CRP, IL-6, FIB and ADRs after the application of DSCIs plus WM. According to NMA results, compared with WM alone, the 7 types of CHIs combined with WM therapy were associated with significantly improved the therapeutic effect. In addition, the results of the multidimensional cluster analysis demonstrated that $\mathrm{DH}+\mathrm{WM}$ and DS+WM had better therapeutic effects. In terms of the ranking of SUCRA probabilities, DH + WM was superior to other CHIs for improving the clinical effectiveness rate. However, since different CHIs have different effects and reactions on patients, the clinical selection of CHIs should better improve the efficacy according to the patient's condition.

$\mathrm{DH}$ is a TCM compound preparation extracted from two famous herbs, Danshen (Radix Salviae Miltiorrhizae, Salvia miltiorrhiza Bunge) and Honghua (Carthami Flos, Carthamus tinctorius L.) (Feng et al., 2020). Since DH launch in 2002, it has 

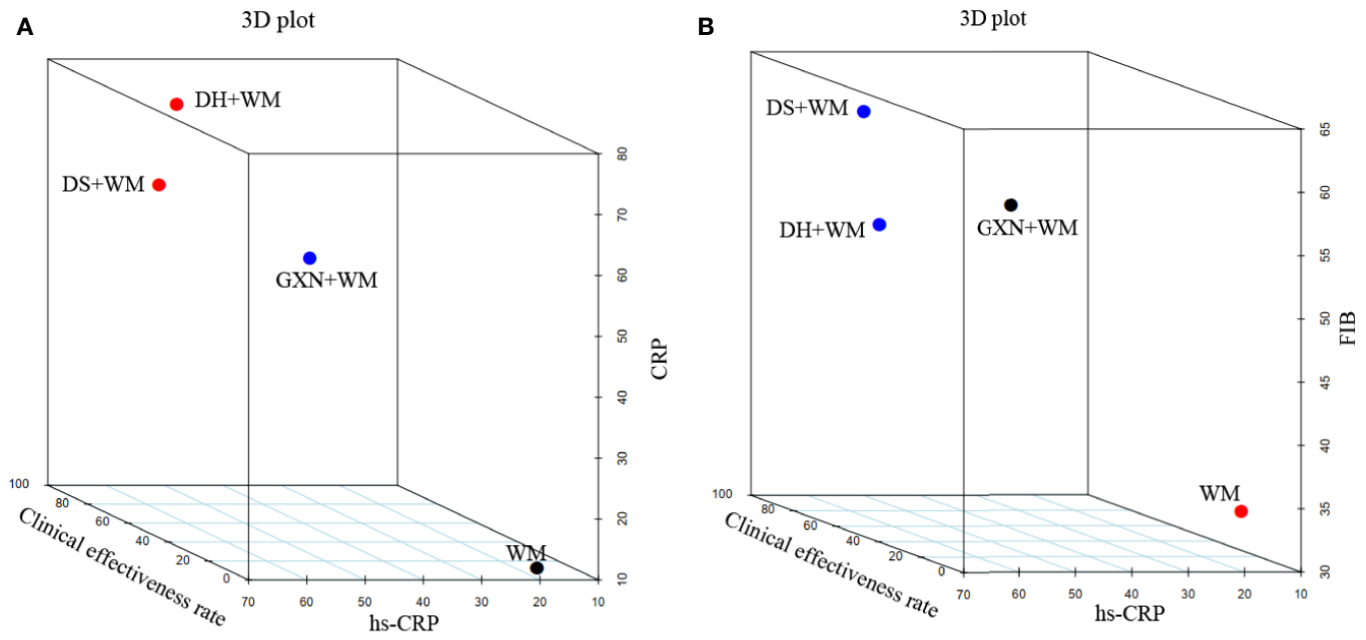

C

3D plot

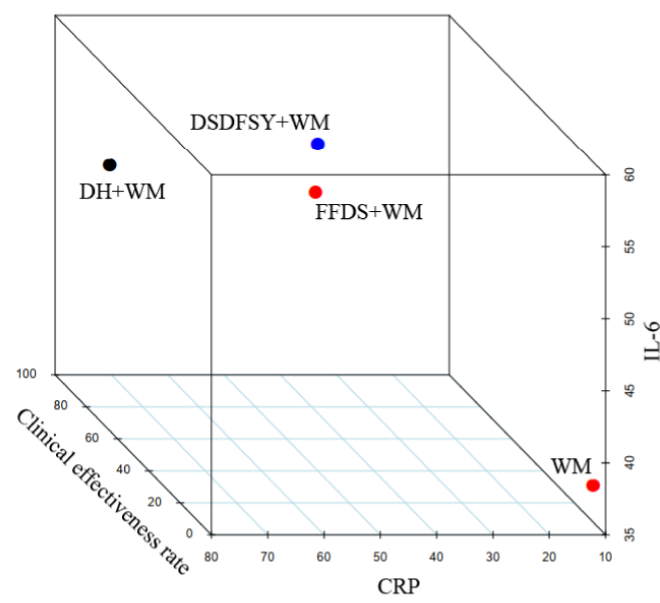

D

3D plot

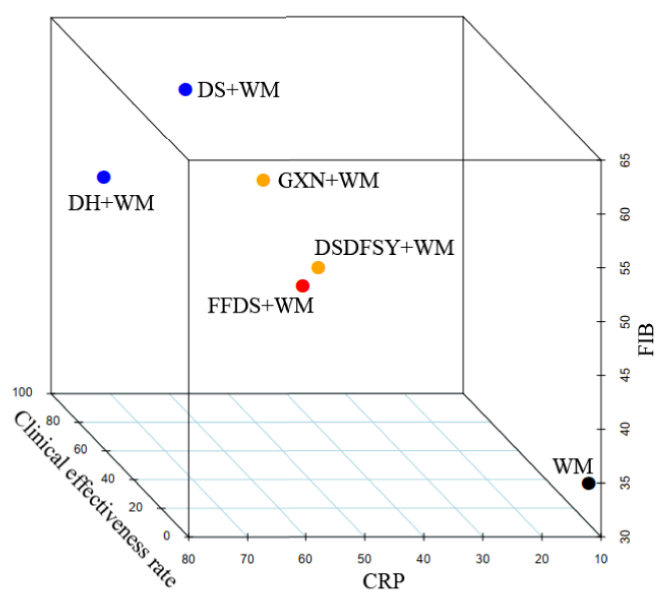

E

3D plot

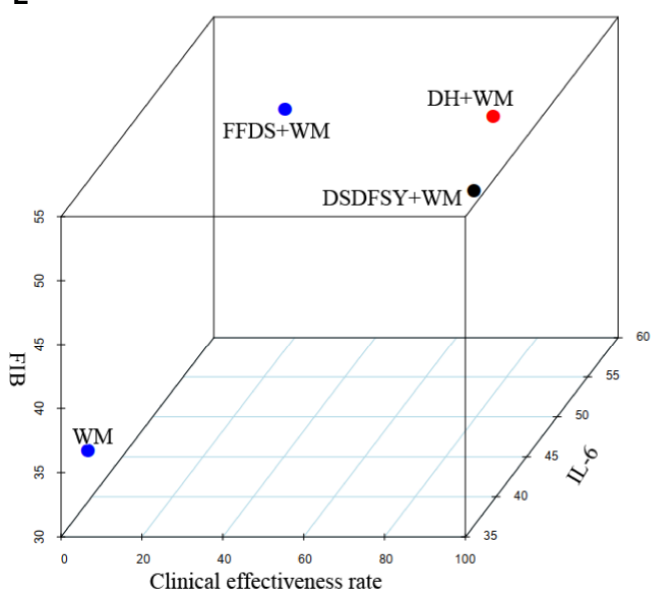

FIGURE 5 | Multidimensional cluster analysis plots. The different colors dots represent different types of interventions. (A) hs-CRP $(X$ axis), clinical effectiveness rate $(Y$ axis) and CRP ( $Z$ axis); (B) hs-CRP $(X$ axis), clinical effectiveness rate $(Y$ axis) and FIB ( $Z$ axis); (C) CRP ( $X$ axis), clinical effectiveness rate $(Y$ axis) and IL-6 ( $Z$ axis); (D) CRP ( $X$ axis), clinical effectiveness rate ( $Y$ axis) and FIB (Z axis); (E) IL-6 (X axis), clinical effectiveness rate ( $Y$ axis) and FIB (Z axis). (DS, Danshen injection; FFDS, Fufang Danshen injection; DH, Danhong injection; DSDFSY, Dansenduofensuanyan injection; DSCXQ, Danshenchuanxiongain injection; STS, Sodium Tanshinone IIA Sulfonate injection; GXN, Guanxinning injection; WM, western medicine; hs-CRP, hypersensitive C-reactive protein; CRP, C-reactive protein; IL-6, interleukin-6; FlB, fibrinogen). 


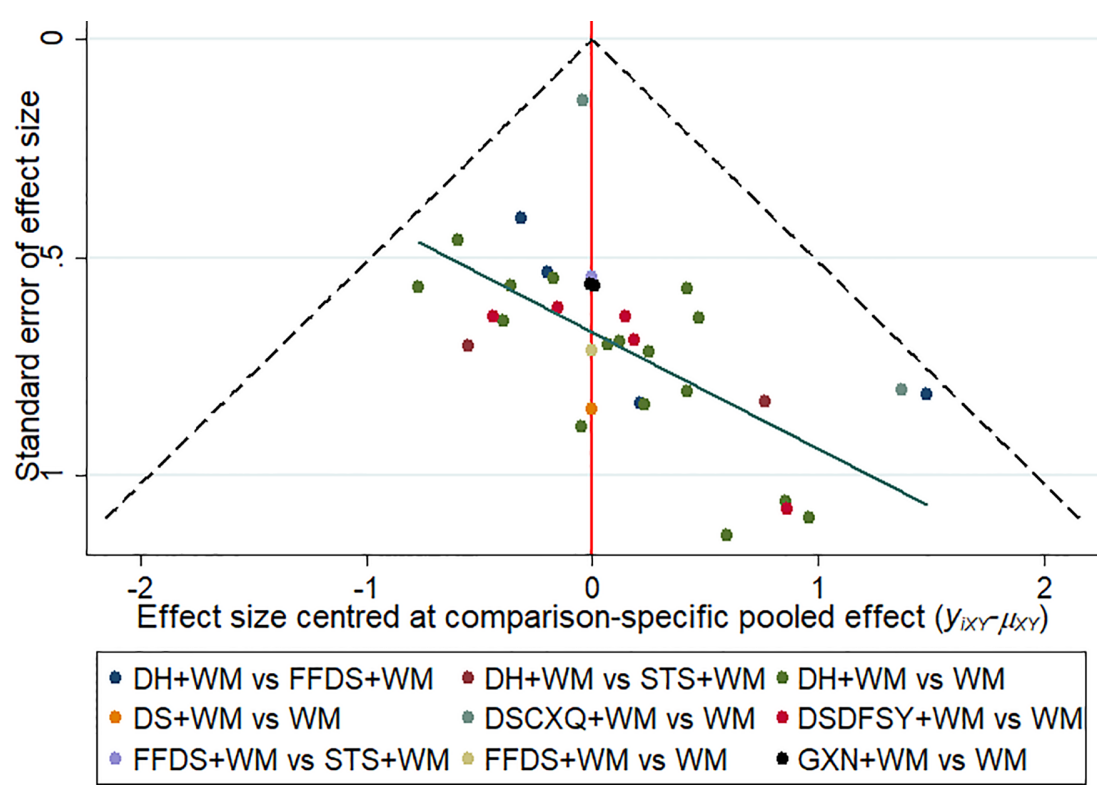

FIGURE 6 | Funnel plots of clinical effectiveness rate. The vertical axis represents "standard error of effect size" and horizontal axis represents "effect size centred at comparison-specific pooled effect ( $\left(\mathrm{y}_{\mathrm{ixy}}-\mu_{\mathrm{xy}}\right)$ ). (DS, Danshen injection; FFDS, Fufang Danshen injection; DH, Danhong injection; DSDFSY, Dansenduofensuanyan injection; DSCXQ, Danshenchuanxiongqin injection; STS, Sodium Tanshinone IIA Sulfonate injection; GXN, Guanxinning injection; WM, western medicine).

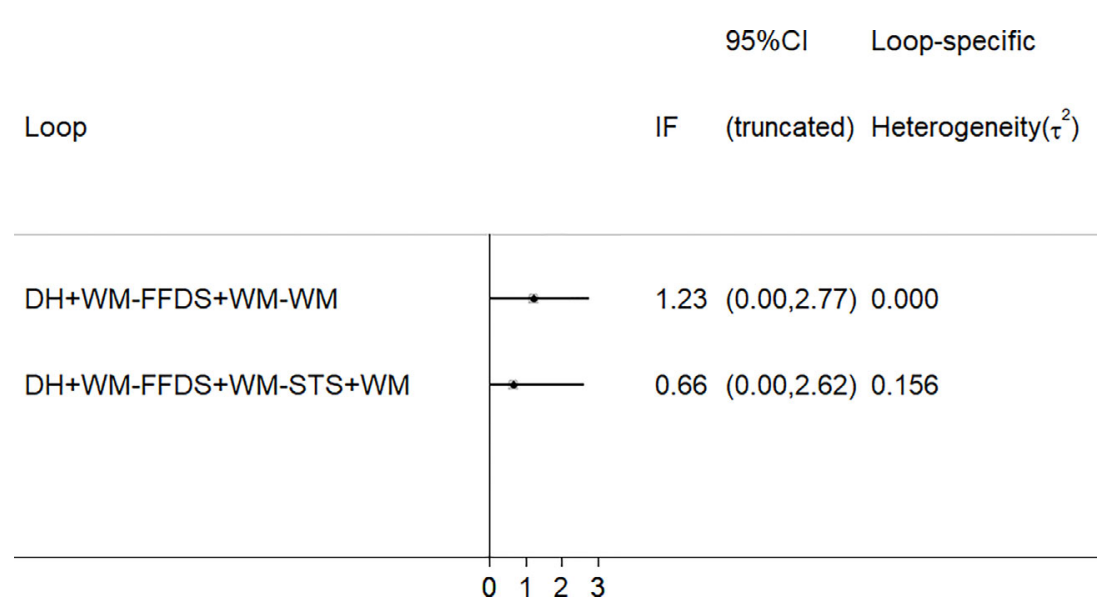

FIGURE 7 | Schematic diagram of consistency. (FFDS, Fufang Danshen injection; DH, Danhong injection; STS, Sodium Tanshinone IIA Sulfonate injection; WM, western medicine).

been widely used to prevent and treat ACS (Yao et al., 2011). The main active ingredients of $\mathrm{DH}$ are flavonoids and phenolic compounds, for example, danshensu, salvianolic acid B, protocatechuic aldehyde, tanshinone IIA, rosmarinic acid and hydroxysafflor yellow A (Xie et al., 2014; Wang et al., 2020). In addition, previous pharmacological studies have demonstrated that DH have the effects of antioxidant, anti-inflammatory, vasodilation and protect cardiac muscle due to its main pharmacological active components (Wan et al., 2011; Jiang et al., 2015). For example, previous studies have demonstrated that salvianolic acid $\mathrm{B}$ could strongly inhibit tumor necrosis factor- $\alpha$-induced nuclear factor- $\kappa B$ (NF- $\kappa B$ ) activation in human aortic endothelial cells (Chen et al., 2001). Furthermore, DH combined with conventional therapy for 2 weeks can significantly reduce the plasma levels of plasma fibrinogen $\mathrm{C}$ (FIB-C), glucose protein II b/III a receptor 
complex and hs-CRP in ACS patients after PCI (Chen et al., 2009; Zhao and Jiang, 2011). In one study, the results indicated that DH + WM not only improved symptoms in patients with UA, but also improved some laboratory indicators, such as CRP, N-terminal brain natriuretic peptide and homocysteine (Sun et al., 2014). Currently, one published NMA showed that $\mathrm{DH}+\mathrm{WM}$ had advantages in improving the total efficacy and electrocardiography of UA (Liu et al., 2018).

DS is one of most commonly used TCM preparation for treating coronary heart disease, heart-stroke and cerebrovascular diseases. Phenolic acids, including danshensu, salvianolic acid A/B, lithospermic acid B and rosmarinic acid, are the main active contents of DS, extracted from Danshen (Zhang et al., 2005). Besides, the most notable effects of phenolic acid in Danshen are antioxidant, anticoagulant, anti-thrombotic and cell protection (Wang et al., 2007; Orgah et al., 2020). A systematic review revealed that DS as one adjuvant treatment of WM for UA could significantly improve the total clinical effectiveness rate. Furthermore, it also could significantly correct T-wave inversion, reduce the level of FIB and adjust blood lipid level (Wu et al., 2017).

Apart from efficacy, the safety of CHIs in the treatment of ACS is also an important issue worthy of consideration by clinicians. In this study, 28 studies did not address ADRs, and 17 studies indicated that there were no significant ADRs. Thus, we could not draw detailed conclusions about the safety information of CHIs. According to the results, the ADRs mainly included allergic rash, vomiting, drowsiness, flushing, mild gastrointestinal reactions and headache. Previous studies had shown that most of the ADRs linked to DHI therapy were mild and moderately severe, with the primary disposition of discontinuation and without other treatment (Li et al., 2015). In addition, it is noteworthy to monitor the ADRs of patients 30 minutes after CHIs injection. Since few studies in this NMA had focused on ADRs. Therefore, more experiments and clinical evidence are needed to verify the safety of these CHIs.

In terms of the design and contents, this NMA study has some particular merits as follows. First, this is the first NMA to evaluate the efficacy and safety of DSCIs plus WM in the treatment of ACS. Second, it strictly established inclusion and exclusion criteria and a comprehensive literature search. Finally, the rankings of eligible CHIs regarding the different outcome indicators provide evidence and recommendation for the clinical selection of medication.

Nevertheless, several potential limitations of the present NMA should be considered. First, all the enrolled RCTs were only performed in Chinese patients, so it is not clear whether the conclusions of this current study are applicable to ACS patients in other countries. Second, the quality of included studies was not high, mainly because most trials did not report detailed information of allocation concealment and blinding. In addition, due to the diversity of WM and the different doses and courses of CHIs, there may have been clinical heterogeneity. For this reason, we believe that the methodological quality of clinical trials should be valued and improved, in order to promote the appropriate use of CHIs. For instance, RCTs should be registered in advance to ensure transparency, and secondly, the test process should be reported in as much detail as possible in the literature. Despite the above limitations, the results of present NMA demonstrated a complete evaluation of the clinical effect in multiple aspects and provided several clinical suggestions of different CHIs for ACS patients.

\section{CONCLUSION}

In summary, this NMA results showed that DSCIs combined with WM therapy could have a positive influence on patients with ACS. What's more, compared with WM alone, DSCIs combined with WM therapy might be associated with significantly improved the therapeutic effect. In addition, $\mathrm{DH}+\mathrm{WM}$ and $\mathrm{DS}+\mathrm{WM}$ therapies are potentially the preferred treatments for ACS. However, since most eligible RCTs studies did not focus on the monitoring of ADRs, the safety of these CHIs needs to be further explored. Due to several limitations, more large samples, high-quality clinical and multicenter RCTs studies should be tested and verified in the future.

\section{DATA AVAILABILITY STATEMENT}

The raw data supporting the conclusions of this article will be made available by the authors, without undue reservation.

\section{AUTHOR CONTRIBUTIONS}

SG and JW conceived and designed the study. SG, MN, SJ, and $\mathrm{JZ}$ conducted the systematic review and extracted and analyzed the data. WZ and XL performed interpretation of results. SG drafted the initial manuscript. MW and XZ critically reviewed the manuscript for important intellectual content. All authors contributed to the article and approved the submitted version.

\section{FUNDING}

This work was supported by the Young Scientists Training Program of Beijing University of Chinese Medicine and the National Nature Science Foundation of China (Grant nos. 81673829).

\section{SUPPLEMENTARY MATERIAL}

The Supplementary Material for this article can be found online at: https://www.frontiersin.org/articles/10.3389/fphar.2020.01260/ full\#supplementary-material 


\section{REFERENCES}

Ahlqvist, E., Storm, P., Käräjämäki, A., Martinell, M., Dorkhan, M., Carlsson, A., et al. (2018). Novel subgroups of adult-onset diabetes and their association with outcomes: a data-driven cluster analysis of six variables. Lancet Diabetes Endocrinol. 6, 361-369. doi: 10.1016/s2213-8587(18)30051-2

Amsterdam, E. A., Wenger, N. K., Brindis, R. G., Casey, D. E.Jr, Ganiats, T. G., Holmes, D. R. Jr., et al. (2014). 2014 AHA/ACC Guideline for the Management of Patients with Non-ST-Elevation Acute Coronary Syndromes: a report of the American College of Cardiology/American Heart Association Task Force on Practice Guidelines. J. Am. Coll. Cardiol. 64, e139-e228. doi: 10.1016/ j.jacc.2014.09.017

Ball, G. H., and Hall, D. J. (1967). A clustering technique for summarizing multivariate data. Behav. Sci. 12, 153-155. doi: 10.1002/bs.3830120210

Buljubasic, N., Akkerhuis, K. M., Cheng, J. M., Oemrawsingh, R. M., GarciaGarcia, H. M., de Boer, S. P., et al. (2017). Fibrinogen in relation to degree and composition of coronary plaque on intravascular ultrasound in patients undergoing coronary angiography. Coron. Artery Dis. 28, 23-32. doi: 10.1097/MCA.0000000000000442

Cai, G., Huang, H. C., Xiao, X., and Pang, L. L. (2011). Effect of Danhong injection on plasma endothelin and C-reactive protein in patients with acute coronary syndrome. Chin. J. Integr. Med. Cardio-Cerebrovasc. 9, 394-395.

Cao, M. Y., Lv, X. H., He, Y., and Liao, K. (2010). The effect of Danhong injection in hemorheology in old patients with acute coronary syndrome. Pract. J. Card. Cereb. Pneumal Vasc. Dis. 18, 541-542.

Centurión, O. A. (2016). Serum biomarkers and source of inflammation in acute coronary syndromes and percutaneous coronary interventions. Cardiovasc. Revasc. Med. 17, 119-128. doi: 10.1016/j.carrev.2016.01.005

Chaimani, A., Higgins, J. P., Mavridis, D., Spyridonos, P., and Salanti, G. (2013). Graphical tools for network meta-analysis in STATA. PloS One 8, e76654. doi: 10.1371/journal.pone.0076654

Chen, K. L. (2012). Clinical research on treating acute coronary syndrome by Danhong injection. Clin. J. Chin. Med. 4, 82-83.

Chen, X. G., and Wang, H. P. (2011). Clinical observation of Danhong injection in the treatment of senile acute coronary syndrome. Chin. J. Clin. Res. 24, 156157.

Chen, Y. H., Lin, S. J., Ku, H. H., Shiao, M. S., Lin, F. Y., Chen, J. W., et al. (2001). Salvianolic acid B attenuates VCAM-1 and ICAM-1 expression in TNF-alphatreated human aortic endothelial cells. J. Cell Biochem. 82, 512-521. doi: $10.1002 /$ jcb. 1176

Chen, Z. Q., Hong, L., and Wang, H. (2009). Effect of danhong injection on platelet activation and inflammatory factors in patients of acute coronary syndrome after intervention therapy. Zhongguo Zhong Xi Yi Jie He Za Zhi 29, 692-694.

Cheng, T. O. (2006). Danshen: a versatile Chinese herbal drug for the treatment of coronary heart disease. Int. J. Cardiol. 113, 437-438. doi: 10.1016/ j.ijcard.2005.10.026

Cope, S., and Jansen, J. P. (2013). Quantitative summaries of treatment effect estimates obtained with network meta-analysis of survival curves to inform decision-making. BMC Med. Res. Methodol. 13, 147. doi: 10.1186/1471-2288-13-147

Crainiceanu, C. M., and Goldsmith, A. J. (2010). Bayesian Functional Data Analysis Using WinBUGS. J. Stat. Software 32, i11.

Cui, S. D. (2008). Clinical observation of 33 cases of acute coronary syndrome treated by Danhong injection. Chin. J. Integr. Med. Cardio-Cerebrovasc. 6, 1091.

de Moerloose, P., Boehlen, F., and Neerman-Arbez, M. (2010). Fibrinogen and the risk of thrombosis. Semin. Thromb. Hemost. 36, 7-17. doi: 10.1055/s-00301248720

Ding, Y. (2012). Effect of Danhong injection on plasma brain natriuretic peptide in patients with acute coronary syndrome. J. Emerg. Tradit. Chin. Med. 21, 281282.

Du, P. (2011). Intervention of Danhong injection on serum MMP-9 in patients with acute coronary syndrome. China Health Ind. 8, 68. doi: 10.16659/ j.cnki.1672-5654.2011.29.052

Du, Z. A., and Chen, J. L. (2009). Clinical observation of Danhong injection in the treatment of acute coronary syndrome. J. Med. Forum. 30, 101-102.

Elhajj, I.II, Haydar, A. A., Hujairi, N. M., and Goldsmith, D. J. (2004). The role of inflammation in acute coronary syndromes: review of the literature. J. Med. Liban. 52, 96-102.
Farooq, V., Gogas, B. D., and Serruys, P. W. (2011). Restenosis: delineating the numerous causes of drug-eluting stent restenosis. Circ. Cardiovasc. Interv. 4, 195-205. doi: 10.1161/CIRCINTERVENTIONS.110.959882

Fei, S. B., Guan, Y. Y., Zhang, L., Lin, G., Zhao, G., Huang, J. F., et al. (2010). Effect of Danhong injection and atorvastatin on the levels of circulating IL -6 , sCD40L and APN in acute coronary syndrome. Chin. J. Integr. Med. CardioCerebrovasc. Dis. 8, 1035-1037.

Feng, C., Wan, H., Zhang, Y., Yu, L., Shao, C., He, Y., et al. (2020). Neuroprotective Effect of Danhong Injection on Cerebral Ischemia-Reperfusion Injury in Rats by Activation of the PI3K-Akt Pathway. Front. Pharmacol. 11, 298. doi: $10.3389 /$ fphar.2020.00298

Feng, Z. H. (2011). The clinical study of Danhong injection on the levels of serum hs-CRP and MMP-9 in patients with acute coronary syndromes. China Health Ind. 8, 16-17. doi: 10.16659/j.cnki.1672-5654.2011.29.048

Grant, R. L. (2019). The uptake of Bayesian methods in biomedical meta-analyses: A scoping revie-2016). J. Evid. Based Med. 12, 69-75. doi: 10.1111/jebm.12326

Gu, Y. F., Zhang, X., Jin, J., Xue, S. F., Chen, J. F., Fan, C. F., et al. (2011). The effect of Danhong injection on serum high sensitivity C-reaction protein in patients with acute coronary syndrome. Chin. J. Cardiovasc. Res. 9, 357-359.

Guan, Y. Y., Fei, S. B., Wang, Y., Sheng, Z. Q., Shi, L. S., Zhang, L., et al. (2010). Effect of Danhong injection on circulating inflammatory factors and TXB2 in acute coronary syndrome. Chin. J. Integr. Med. Cardio-Cerebrovasc. Dis. 8, 1039-1040.

Haldar, P., Pavord, I. D., Shaw, D. E., Berry, M. A., Thomas, M., Brightling, C. E., et al. (2008). Cluster Analysis and Clinical Asthma Phenotypes. Am. J. Respir. Crit. Care Med. 178, 218-224. doi: 10.1164/rccm.200711-1754oc

He, Y. L. (2014). Clinical analysis of Danhong injection applied to treat acute coronary artery syndrome. J. Sichuan Tradit. Chin. Med. 32, 165-167.

Hedayati, T., Yadav, N., and Khanagavi, J. (2018). Non-ST-Segment Acute Coronary Syndromes. Cardiol. Clin. 36, 37-52. doi: 10.1016/j.ccl.2017.08.003

Higgins, J. P., Altman, D. G., Gøtzsche, P. C., Jüni, P., Moher, D., Oxman, A. D., et al. (2011). The Cochrane Collaboration's tool for assessing risk of bias in randomised trials. BMJ 343, d5928. doi: 10.1136/bmj.d5928

Hong, Y. D., Wu, H., Mo, H. H., and Li, F. Y. (2004). Effects of Xiangdan injection in acute coronary syndrome and inflammatory markers. Tradit. Chin. Drug Res. Clin. Pharmacol. 15, 425-428. doi: 10.19378/j.issn.1003-9783.2004.06.019

Hou, X. J. (2013). Comparative observation of the efficacy of Danhong injection and Tanshinone IIa sulfonate injection in the treatment of 99 cases of acute coronary syndrome. Asia-Pacific Tradit. Med. 9, 163-164.

Huang, X., Duan, X., Zhu, Y., Wang, K., Wu, J., and Tian, X. (2019). Comparative efficacy of Chinese herbal injections for the treatment of community-acquired pneumonia: A Bayesian network meta-analysis of randomized controlled trials. Phytomedicine 63, 153009. doi: 10.1016/j.phymed.2019.153009

Hutton, B., Salanti, G., Caldwell, D. M., Chaimani, A., Schmid, C. H., Cameron, C., et al. (2015). The PRISMA extension statement for reporting of systematic reviews incorporating network meta-analyses of health care interventions: checklist and explanations. Ann. Intern. Med. 162, 777-784. doi: 10.7326/M14-2385

Jiang, X., Lv, B., Li, P., Ma, X., Wang, T., Zhou, Q., et al. (2015). Bioactivityintegrated UPLC/Q-TOF-MS of Danhong injection to identify NF- $\mathrm{KB}$ inhibitors and anti-inflammatory targets based on endothelial cell culture and network pharmacology. J. Ethnopharmacol. 174, 270-276. doi: 10.1016/ j.jep.2015.08.026

Kirtane, A. J., Sandhu, P., Mehran, R., McEntegart, M., Cristea, E., Brener, S. J., et al. (2014). Association between intraprocedural thrombotic events and adverse outcomes after primary percutaneous coronary intervention for ST-segment elevation myocardial infarction (a Harmonizing Outcomes with RevasculariZatiON and Stents in Acute Myocardial Infarction [HORIZONS-AMI] Substudy). Am. J. Cardiol. 113, 36-43. doi: 10.1016/j.amjcard.2013.08.034

Kosuge, M., and Kimura, K. (2016). Implications of using the Cabrera sequence for diagnosing acute coronary syndrome. Circ. J. 80, 1087-1096. doi: 10.1253/ circj.CJ-16-0126

Kurtul, A., Yarlioglues, M., Murat, S. N., Duran, M., Oksuz, F., Koseoglu, C., et al. (2016). The association of plasma fibrinogen with the extent and complexity of coronary lesions in patients with acute coronary syndrome. Kardiol. Pol. 74, 338-345. doi: 10.5603/KP.a2015.0196

Lao, G. H., Chen, G. Y., Li, H. D., and Chen, J. (2013). Lipid-lowering effect of Danhong injection combined with low-dose simvastatin on acute coronary syndrome. Pract. Clin. Med. 14, 10-11. 
Li, A. X., Jin, M. H., Li, D., and Li, Z. (2019). Clinical effect of Danshen polyphenolate injection combined with ticagrelor in the treatment of patients with acute coronary syndrome. Heilongjiang Med. Pharm. 42, 51-52.

Li, C. L., Huang, H. G., and Deng, P. (2007). Effect of Danhong injection on plasma tissue factors in patients with acute coronary syndrome. J. Chin. Physician. 9, 984-985.

Li, J. H. (2013). Salvia miltiorrhiza polyphenolate injection combined with low molecular weight heparin calcium in the treatment of 46 cases of acute coronary syndrome. Nei Mongol J. Tradit. Chin. Med. 32, 2-3. doi: 10.16040/ j.cnki.cn15-1101.2013.26.158

Li, J. P., Liu, Y., Guo, J. M., Shang, E. X., Zhu, Z. H., Zhu, K. Y., et al. (2017). A Comprehensive Strategy to Evaluate Compatible Stability of Chinese Medicine Injection and Infusion Solutions Based on Chemical Analysis and Bioactivity Assay. Front. Pharmacol. 8, 833. doi: 10.3389/fphar.2017.00833

Li, M., Chen, X. C., Song, W. D., Wang, X. Y., Wei, D. Y., Qin, H. Q., et al. (2016). Effect of Guanxining Injection on serum YKL-40 and hs-CRP levels in patients with non-ST-segment elevation acute coronary syndrome. J. North Pharm. 13, 142-143.

Li, S. P. (2015). Clinical observation of Danhong injection in the treatment of senile acute coronary syndrome. Med. Forum. 19, 1328-1329.

Li, W. H., Zhang, Y. B., Zhu, H., Xu, T. D., Wang, Z. R., Qian, W. H., et al. (2010). Effect of Danhong injection on the high-sensitivity C-reactive protein, fibrinogen and von willebrand factor in patients with acute coronary syndrome. Chin. J. Gen. Pract. 8, 1222-1223. doi: 10.16766/j.cnki.issn.16744152.2010.10.010

Li, X. L., Tang, J. F., Li, W. X., Li, C. X., Zhao, T., Zhao, B. C., et al. (2015). Postmarketing Safety Surveillance and Reevaluation of Danhong Injection: Clinical Study of 30888 Cases. Evid. Based Complement. Alternat. Med. 2015, 610846. doi: $10.1155 / 2015 / 610846$

Li, Y. H., and Ge, H. Y. (2011). Observation on treatment of acute coronary syndrome by Danhong injection. J. Med. Forum. 32, 150-152.

Li, Y. L., and Meng, Z. H. (2008). Danhong injection was used to treat 48 cases of acute coronary syndrome. J. Pract. Tradit. Chin. Intern. Med. 22, 20-21. doi: 10.13729/j.issn.1671-7813.2008.03.010

Li, Y. M., Chen, X. H., Li, J., Hu, J. P., Bian, Y. L., and Wu, Y. J. (2007). Clinical study of Danshen injection in treating acute coronary syndrome. Chin. J. Tradit. Med. Sci. Tech. 14, 392-393.

Li, Y. N., and Song, X. (2011). Effect of Danhong injection on serum hypersensitive C-reactive protein in patients with acute coronary syndrome. Chin. J. Mod. Drug Appl. 5, 167-168. doi: 10.14164/j.cnki.cn11-5581/r.2011.03.018

Liang, J., Zhang, Z. L., Wang, Z. J., and Zhang, K. C. (2019). Effects of Danhong injection on inflammatory factors vascular endothelial function and platelet activation in patients with acute coronary syndrome. Chin. J. Integr. Med. Cardio-Cerebrovasc. Dis. 17, 1999-2001.

Liao, Y. J., Liu, C. H., Ping, Z. X., Jiang, T., and Yao, J. (2013). Effect of Guanxining injection on plasma fibrinogen and C-reactive protein in patients with acute coronary syndrome. Guide China Med. 11, 698-699.

Lin, S. L. (2015). Effect of Salvia Miltiorrhiza Polyphenolate on the level of modified albumin in angina pectoris. Chin. J. Urban Rural Enterp. Hyg. 30, 110-111. doi: 10.16286/j.1003-5052.2015.02.047

Lin, Y. C., and Yu, Z. S. (2017). Influence and its efficacy of salvianolate adjuvant therapy on vascular endothelial function in patients with acute coronary syndrome China Mod. Doct. 55, 26-28.

Liu, S., Wang, K., Duan, X., Wu, J., Zhang, D., Liu, X., et al. (2019). Efficacy of Danshen Class Injection in the Treatment of Acute Cerebral Infarction: A Bayesian Network Meta-Analysis of Randomized Controlled Trials. Evid. Based Complement. Alternat. Med. 2019, 5814749. doi: 10.1155/2019/5814749

Liu, S., Wu, J., Zhang, D., and Tan, D. (2018). What are the best Salvia miltiorrhiza injection classes for treatment of unstable angina pectoris? A systematic review and network Meta-analysis. J. Tradit. Chin. Med. 38, 321-338. doi: 10.1016/ S0254-6272(18)30623-X

Liu, X., Wang, Y., and Jin, J. (2008). Clinical analysis of 300 cases of acute coronary syndrome treated by Danhong injection. Pract. J. Card. Cereb. Pneumal Vasc. Dis. 16, 24-25.

Liuzzo, G. (2001). Atherosclerosis: an inflammatory disease. Rays 26, 221-230.

Long, T. X. (2011). Study on the clinical efficacy of Danshen injection in the treatment of acute coronary syndrome. Chin. J. Clin. Ration. Drug Use 4, 4445. doi: 10.15887/j.cnki.13-1389/r.2011.27.006
Ma, J., and Liang, X. Y. (2016). Effect of Danshen injection on serum homocysteine, folic acid and C-reactive protein levels in patients with STsegment elevation acute myocardial infarction. Chin. J. Integr. Med. CardioCerebrovasc. Dis. 14, 2528-2530.

Meim, X. D., Cao, Y. F., Che, Y. Y., Li, J., Shang, Z. P., Zhao, W. J., et al. (2019). Danshen: a phytochemical and pharmacological overview. Chin. J. Nat. Med. 17, 59-80. doi: 10.1016/S1875-5364(19)30010-X

Ministry of Health of People's Republic of China (1993). Guidelines of TCM Clinical Research. 1st ed (Beijing: People's Health Publishing House).

Nanchen, D., Klingenberg, R., Gencer, B., Räber, L., Carballo, D., von Eckardstein, A., et al. (2019). Inflammation during acute coronary syndromes - Risk of cardiovascular events and bleeding. Int. J. Cardiol. 287, 13-18. doi: 10.1016/ j.ijcard.2019.03.049

Nie, L. S., and Zhao, N. J. (2012). Clinical observations of 60 patients regarding the effect of danhong injection on hs-CRP of patients with acute coronary syndrome. World Health Dig. 9, 409-410. doi: 10.3969/j.issn.16725085.2012.16.397

Orgah, J. O., He, S., Wang, Y., Jiang, M., Wang, Y., Orgah, E. A., et al. (2020). Pharmacological potential of the combination of Salvia miltiorrhiza (Danshen) and Carthamus tinctorius (Honghua) for diabetes mellitus and its cardiovascular complications. Pharmacol. Res. 153, 104654. doi: 10.1016/ j.phrs.2020.104654

Pan, B., Wu, Y., Yang, Q., Ge, L., Gao, C., Xun, Y., et al. (2019). The impact of major dietary patterns on glycemic control, cardiovascular risk factors, and weight loss in patients with type 2 diabetes: A network meta-analysis. J. Evid. Based Med. 12, 29-39. doi: 10.1111/jebm.12312

Riley, R. D., Higgins, J. P., and Deeks, J. J. (2011). Interpretation of random effects meta-analyses. BMJ 342, d549. doi: 10.1136/bmj.d549

Rouse, B., Chaimani, A., and Li, T. (2017). Network meta-analysis: an introduction for clinicians. Intern. Emerg. Med. 12, 103-111. doi: 10.1007/s11739-016-1583-7

Ruan, L., Jiao, X. M., Li, J., Wang, C. L., and Li, T. (2017). Effects of Xiangdan injection on serum levels of $\mathrm{C}$-reactive protein, amino terminal brain natriuretic peptide and cardiac enzymes of patients with acute coronary syndrome. Prog. Mod. Biomed. 17, 3481-3485. doi: 10.13241/j.cnki.pmb.2017.18.018

Saremi, F. (2017). Cardiac MR imaging in acute coronary syndrome: application and image interpretation. Radiology 282, 17-32. doi: 10.1148/radiol.2016152849

Shi, K. (2013). Treating cute coronary syndrome with Danshen. Clin. J. Chin. Med. $5,23-24$

Stefanadis, C., Diamantopoulos, L., Dernellis, J., Economou, E., Tsiamis, E., Toutouzas, K., et al. (2000). Heat production of atherosclerotic plaques and inflammation assessed by the acute phase proteins in acute coronary syndromes. J. Mol. Cell Cardiol. 32, 43-52. doi: 10.1006/jmcc.1999.1049

$\mathrm{Su}, \mathrm{H}$. C., and Zhao, C. (2017). Effect of Danshen injection on serum homocysteine and $\mathrm{C}$ reactive protein in patients with ST segment elevation acute myocardial infarction. J. Snake. 29, 415-416.

Su, K., and Jiang, Y. B. (2012). Effect of Danhong injection on serum high sensitivity C-reactive protein in patients with acute coronary syndrome. China Pract. Med. 7, 22-23. doi: 10.14163/j.cnki.11-5547/r.2012.25.095

Sun, K., Fu, C., Nie, S., and You, Y. (2014). The index and improvement effect of using Danhong injection to patients with atherosclerosis symptoms of coronary heart disease (CHD). Pak. J. Pharm. Sci. 27, 1699-1704.

Sun, H., Li, Y., Su, Y., Wu, X., Zhou, X., Han, J., et al. (2019). Efficacy and safety of anti-EGFR monoclonal antibodies combined with different chemotherapy regimens in patients with RAS wild-type metastatic colorectal cancer: A meta-analysis. J. Evid. Based Med. 12, 300-312. doi: 10.1111/jebm.12360

Tan, D., Wu, J. R., Zhang, X. M., Liu, S., and Zhang, B. (2018). Sodium Tanshinone II A Sulfonate Injection as Adjuvant Treatment for Unstable Angina Pectoris: A Meta-Analysis of 17 Randomized Controlled Trials. Chin. J. Integr. Med. 24, 156-160. doi: 10.1007/s11655-017-2424-x

Timmis, A. (2015). Acute coronary syndromes. BMJ 351, h5153. doi: 10.1136/ bmj.h5153

Trinquart, L., Attiche, N., Bafeta, A., Porcher, R., and Ravaud, P. (2016). Uncertainty in Treatment Rankings: Reanalysis of Network Meta-analyses of Randomized Trials. Ann. Intern. Med. 164, 666-673. doi: 10.7326/M15-2521

Virani, S. S., Alonso, A., Benjamin, E. J., Bittencourt, M. S., Callaway, C. W., Carson, A. P., et al. (2020). Heart Disease and Stroke Statistics-2020 Update: A Report From the American Heart Association. Circulation 141, e139-e596. doi: 10.1161/CIR.0000000000000757 
Wan, L. H., Chen, J., Li, L., Xiong, W. B., and Zhou, L. M. (2011). Protective effects of Carthamus tinctorius injection on isoprenaline-induced myocardial injury in rats. Pharm. Biol. 49, 1204-1209. doi: 10.3109/ 13880209.2011.576348

Wang, D., Yang, X. H., Zhang, J. D., Li, R. B., Jia, M., and Cui, X. R. (2018). Compared efficacy of clopidogrel and ticagrelor in treating acute coronary syndrome: a meta-analysis. BMC Cardiovasc. Disord. 18, 217. doi: 10.1186/ s12872-018-0948-4

Wang, J. X., Xie, Y. Q., Guo, C. F., Gao, H. Y., Peng, H., Chen, Y. Q., et al. (2015). Clinical observation of 245 cases of elderly patients with acute coronary syndrome treated by Danhong injection. World Chin. Med. 10, 1.

Wang, S., L1, Yu, Sun, G., Liu, Y., Hu, W., Liu, Y., et al. (2020). Danhong Injection Protects Hemorrhagic Brain by Increasing Peroxiredoxin 1 in Aged Rats. Front. Pharmacol. 11, 346. doi: 10.3389/fphar.2020.00346

Wang, S. P., and Zhang, A. P. (2012). Clinical observation of Danhong injection in treating senile acute coronary syndrome. J. Emerg. Tradit. Chin. Med. 21, 1181-1182.

Wang, X., Morris-Natschke, S. L., and Lee, K. H. (2007). New developments in the chemistry and biology of the bioactive constituents of Tanshen. Med. Res. Rev. 27, 133-148. doi: 10.1002/med.20077

Wei, Y. S. (2012). Effect of Danhong injection on plasma endothelin-1 and high sensitivity C-reactive protein in patients with acute coronary syndrome. China Mod. Med. 19, 31-32.

Wu, G. P., Huang, X. H., and Yao, Z. (2009). Influence of danshen ligustrazin for injection on serum high-sensitivity C-reactive protein and cardiac function of patients with acute coronary syndrome. J. Hainan Med. Uni. 15, 858-860. doi: 10.13210/j.cnki.jhmu.2009.08.004

Wu, J. R., Zhang, X. M., and Zhang, B. (2015). Danhong injection in the treatment of acute coronary syndrome: a systematic review and meta-analysis. Am. J. Chin. Med. 43, 199-214. doi: 10.1142/S0192415X15500135

Wu, J. R., Liu, S., Zhang, X. M., and Zhang, B. (2017). Danshen injection as adjuvant treatment for unstable angina pectoris: A systematic review and meta-analysis. Chin. J. Integr. Med. 23, 306-311. doi: 10.1007/s11655-0162272-0

Xia, L. J., Chen, W. D., and Wu, Y. M. (2017). Clinical observation on curative effect of acute coronary syndrome treated with salvianolate injection and its influences on serum hs-CRP, IL-6 levels. Chin. J. Tradit. Med. Sci. Tech. $24,4-5$.

Xia, S. L., and Wang, Y. (2011). Effects of Guanxinning injection on non-STsegment elevation acute coronary syndrome. China Mod. Doct. 49, 99-100.

Xiao, M. S. (2009). Comparision of the curative effects of danhong injection VS compound danshen injection in the treatment for the elderly patients with non-ST-elevation acute coronary syndrome. J. Pract. Electrocardiol. 18, 19-21. doi: 10.13308/j.issn.1008-0740.2009.01.003

Xie, Y. Y., Xiao, X., Luo, J. M., Fu, C., Wang, Q. W., Wang, Y. M., et al. (2014). Integrating qualitative and quantitative characterization of traditional Chinese medicine injection by high-performance liquid chromatography with diode array detection and tandem mass spectrometry. J. Sep. Sci. 37, 1438-1447. doi: $10.1002 /$ jssc. 201400129

$\mathrm{Xu}, \mathrm{L}$. C. (2011). Clinical observation of 42 cases of acute coronary syndrome treated by Danhong injection combined with western medicine. Nei Mongol J. Tradit. Chin. Med. 30, 71-72. doi: 10.16040/j.cnki.cn15-1101.2011.21.123

Yang, L. J. (2010). Effect of Danhong injection on plasma endothelin in patients with acute coronary syndrome. Mod. J. Integr. Tradit. Chin. West. Med. 19, 51-52.

Yang, M. J., and Zhang, B. S. (2010). Cost-effectiveness analysis of two regimens for acute coronary syndrome. Ningxia Med. J. 32, 1252-1253. doi: 10.13621/ j.1001-5949.2010.12.037

Yao, J. Y., Zhi, M., Cao, W. T., Huang, Y., and Li, C. J. (2011). Successful treatment with danhong injection for hepatic veno-occlusive disease. Hepatogastroenterology 58, 992-995.
Yuan, J. F., Hu, J. W., Chen, Z. Y., and Wang, D. H. (2020). Screening of cardioprotective diseases bioactive components from Danshen extracts and LC-MS analysis. Biomed. Chromatogr. 34, e4823. doi: 10.1002/bmc.4823

Zhang, D., Wu, J., Duan, X., Wang, K., Ni, M., Liu, S., et al. (2019). Network MetaAnalysis of Chinese Herbal Injections Plus the FOLFOX Regimen for the Treatment of Colorectal Cancer in China. Integr. Cancer Ther. 18, 1534735419827098. doi: 10.1177/1534735419827098

Zhang, D. W., Wang, S. L., Wang, P. L., Du, J. P., Gao, Z. Y., Wang, C. L., et al. (2020). The efficacy of Chinese herbal medicines on acute coronary syndrome with renal insufficiency after percutaneous coronary intervention. J. Ethnopharmacol. 248, 112354. doi: 10.1016/j.jep.2019.112354

Zhang, J. L., Cui, M., He, Y., Yu, H. L., and Guo, D. A. (2005). Chemical fingerprint and metabolic fingerprint analysis of Danshen injection by HPLC-UV and HPLC-MS methods. J. Pharm. Biomed. Anal. 36, 1029-1035. doi: 10.1016/ j.jpba.2004.09.009

Zhang, S., Shi, P., and Ma, L. (2014). Evaluation of Danshen ligustrazine on improving the clinical symptoms of non-ST elevation acute coronary syndrome (middle and low risk group). China Pract. Med. 9, 143-145. doi: 10.14163/j.cnki.11-5547/r.2014.25.098

Zhang, S. G., He, L. M., Liu, S. Y., Li, Q. Y., and Tan, R. R. (2012). Clinical observation on efficacy and safety of traditional Chinese medicine for activating blood circulation and removing blood stasis in acute coronary syndrome. China \& Foreign Med. Treat. 31, 4-6. doi: 10.16662/j.cnki.16740742.2012.13.011

Zhang, X., Wu, J., Zhang, B., and Zhou, W. (2016). Danshenchuanxiongqin injection in the treatment of unstable angina pectoris: a systematic review and Meta-analysis. J. Tradit. Chin. Med. 36, 144-150. doi: 10.1016/s0254-6272(16) 30020-6

Zhang, Z. C., Chang, X. G., Xu, Y., and Zhang, L. Q. (2010). Effects of DANHONG injection on hs-CRP, IL6, TC, TG and LDL-C in old patients with acute coronary syndrome. Chin. Arch. Tradit. Chin. Med. 28, 2237-2238. doi: 10.13193/j.archtcm.2010.10.223.zhangzhch.033

Zhao, P. X., and Jiang, S. (2011). Effect of danhong injection on ET-1, sP-sel, and hs-CRP in patients with acute coronary syndrome undergoing percutaneous coronary intervention. Zhongguo Zhong Xi Yi Jie He Za Zh 31, 11-14.

Zhao, Q. F. (2011). Observation on the curative effect of Guanxining injection in treating acute coronary syndrome. Chin. J. Misdiagnostics. 11, 7864-7865.

Zhao, S., Tang, Y., Cai, H., Liu, W., Zhang, L., Chen, D., et al. (2018). Treatment of Danhong Injection Combined with Naoxintong Capsule in Acute Coronary Syndrome Patients Undergoing PCI Operation: Study for a Randomized Controlled and Double-Blind Trial. Evid. Based Complement. Alternat. Med. 2018, 8485472. doi: 10.1155/2018/8485472

Zhou, K. J. (2011). The effect of Danhong injection on the level of serum hs-CRP in patients with acute coronary syndromes. China Health Ind. 8, 11-12. doi: 10.16659/j.cnki.1672-5654.2011.29.046

Zou, J. B., Zhang, X. F., Wang, J., Wang, F., Cheng, J. X., Yang, F. Y., et al. (2018). The Therapeutic Efficacy of Danhong Injection Combined with Percutaneous Coronary Intervention in Acute Coronary Syndrome: A Systematic Review and Meta-Analysis. Front. Pharmacol. 9, 550. doi: 10.3389/fphar.2018.00550

Conflict of Interest: The authors declare that the research was conducted in the absence of any commercial or financial relationships that could be construed as a potential conflict of interest.

Copyright (C) 2020 Guo, Wu, Ni, Jia, Zhang, Zhou, Liu, Wang and Zhang. This is an open-access article distributed under the terms of the Creative Commons Attribution License (CC BY). The use, distribution or reproduction in other forums is permitted, provided the original author(s) and the copyright owner(s) are credited and that the original publication in this journal is cited, in accordance with accepted academic practice. No use, distribution or reproduction is permitted which does not comply with these terms. 